Rev. Int. Contam. Ambie. 36 (3) 497-515, 2020

https://doi.org/10.20937/RICA.53185

\title{
REMOCIÓN DE CONTAMINANTES DEL NEJAYOTE CON ALGINATO Y QUITOSANO
}

\author{
Removal of nejayote contaminants with alginate and chitosan
}

\author{
Karina Jannet VACIO-MURO ${ }^{1}$, Juan Antonio LOZANO-ÁLVAREZ ${ }^{1}$, \\ Mónica Noel SÁNCHEZ-GONZÁLEZ², Norma Angélica CHÁVEZ VELA ${ }^{1}$, \\ Eduardo TORRES-RAMÍREZ ${ }^{3}$ y Juan JÁUREGUI-RINCÓN ${ }^{1 *}$
}

${ }^{1}$ Departamento de Ingeniería Bioquímica, Universidad Autónoma de Aguascalientes. Av. Universidad $940 \mathrm{Cd}$. Universitaria, C.P. 20131 Aguascalientes, Aguascalientes México

${ }^{2}$ Facultad de Ingeniería Química, Universidad Autónoma de Yucatán, Periférico Norte, kilómetro 33.5 Tablaje Catastral 13615, Chuburná de Hidalgo Inn, C.P. 97203 Mérida, Yucatán

${ }^{3}$ Instituto de Ciencias, Benemérita Universidad Autónoma de Puebla. Edificio 103-E, Ciudad Universitaria, colonia San Manuel, C.P. 72570 Puebla, México

*Autor para correspondencia: jjaureg@correo.uaa.mx

(Recibido marzo 2018, aceptado noviembre 2019)

Palabras clave: nixtamalización, biopolímeros, tratamiento de aguas, materia orgánica

\section{RESUMEN}

El nejayote es el agua residual proveniente del proceso de nixtamalización que se caracteriza por poseer un $\mathrm{pH}$ alcalino $(\mathrm{pH}>10)$ así como altas concentraciones de materia orgánica disuelta y suspendida (DQO $>10200 \mathrm{mg} / \mathrm{L}$ ), por lo que se considera uno de los efluentes más contaminantes vertidos a diferentes cuerpos de agua. Se probó la efectividad del alginato de sodio y el quitosano para disminuir los contaminantes del nejayote. La aplicación de alginato de sodio involucró la adsorción y gelificación con la que se atrapó material suspendido y disuelto, y el uso del quitosano implicó adsorción y precipitación. Se realizó la aplicación de estos polímeros de manera separada y secuencial. En el tratamiento independiente, el alginato resultó más eficiente que el quitosano, obteniéndose una remoción de DQO de $61.43 \pm 0.24 \%$ y de $59.74 \pm 0.36 \%$, respectivamente. En el tratamiento secuencial, la eficiencia de remoción de la DQO con el tratamiento alginato-quitosano resultó ser mayor $(70.19 \pm 0.85 \%)$ que la del tratamiento quitosano-alginato $(67.21 \pm 0.24 \%)$. Por su parte, la cantidad de sólidos totales presentes después del procesamiento se encuentra dentro del límite máximo permisible establecido en las normas oficiales mexicanas NOM-002-SEMARNAT-1996 y NOM-001-SEMARNAT-1996, permitiendo su descarga al sistema de alcantarillado urbano o municipal e incluso puede ser utilizada en suelo para riego agrícola. El uso de estos polímeros biodegradables es una opción viable que tiene potencial para escalarse a nivel industrial, contribuyendo a la depuración de descargas de la industria de la nixtamalización y en beneficio del ambiente.

Key words: nixtamalization, biopolymers, water treatment, organic material

\begin{abstract}
Nejayote is the residual water from the nixtamalization process characterized by an alkaline $\mathrm{pH}(\mathrm{pH}>10)$ as well as high concentrations of dissolved and suspended
\end{abstract}


organic matter (COD > $10200 \mathrm{mg} / \mathrm{L}$ ). As for the above mentioned, nejayote is considered within the more pollutants liquid effluents discharged into different water bodies. In this work, the effectiveness of sodium alginate and chitosan to reduce nejayote pollutants was tested. The application of alginate involved adsorption and gelation, which trapped suspended and dissolved material, and the use of chitosan implied an adsorption-precipitation phenomena. These polymers were used separately and sequentially. In the independent treatments, the alginate was more efficient than the chitosan, obtaining a COD removal of $61.43 \pm 0.24 \%$ and of $59.74 \pm 0.36 \%$ respectively. In sequential treatments, the removal efficiency of COD with alginate-chitosan was higher $(70.19 \pm 0.85 \%)$ than that of the chitosan-alginate treatment $(67.21 \pm 0.24 \%)$. On the other hand, the reduction in the amount of total solids allows that the effluent, after the processing, is within the maximum permissible limit established by the official Mexican standards NOM-002-SEMARNAT-1996 and NOM-001-SEMARNAT-1996.,This permits its discharge to urban or municipal sewerage systems and it can even be used for agricultural irrigation. The use of these biodegradable polymers is a viable option that has the potential to scale at the industrial level, contributing to the purification of discharges from the nixtamalization industry and to the benefit of the environment.

\section{INTRODUCCIÓN}

El maíz es un producto del campo mexicano muy importante ya que desde hace más de 3500 años forma parte primordial de la dieta de los mexicanos (Gutiérrez-Cortéz et al. 2010, Ferreira-Rolón et al. 2014). Adicionalmente, debido a la globalización, el maíz también es un producto altamente consumido en lugares como los Estados Unidos de América, Colombia, Venezuela, Brasil y China, entre otros (Janve et al. 2013, García-Zamora et al. 2015, SuárezMeraz et al. 2016).

La elaboración de los distintos productos de maíz se efectúa con el producto resultante de su tratamiento alcalino, proceso denominado nixtamalización, el cual consta de cuatro pasos: 1) mezcla de maíz, agua e hidróxido de calcio; 2) cocimiento durante 50 a 90 min; 3) remojo del maíz en el agua de cocción por un periodo de 14 a $18 \mathrm{~h}$ y 4) recuperación y lavado del maíz para remover el exceso de calcio y materia orgánica (Ruiz-Gutiérrez et al. 2010, Janve et al. 2013, Méndez-Albores et al. 2014). El residuo de este proceso contiene pericarpio de maíz, nutrientes como arabinoxilanos, almidón, dextrinas, proteínas, iones de calcio (II), además de incluir compuestos fenólicos antioxidantes como los ácidos ferúlico y cumárico. Desafortunadamente, estos componentes al ser descargados a los ecosistemas acuáticos agotan el oxígeno disuelto representando un impacto ambiental importante para dichos ecosistemas. Esto se ve traducido en valores elevados de la demanda química de oxígeno (DQO = 1670 a $21280 \mathrm{mg} / \mathrm{L}$ ) y demanda bioquímica de oxígeno $\left(\mathrm{DBO}_{5}=190 \mathrm{a}\right.$ $7875 \mathrm{mg} / \mathrm{L}$ ), además de una alta alcalinidad total
(180 a $3260 \mathrm{mg}$ de $\mathrm{CaCO}_{3} / \mathrm{L}$ ) como lo han reportado Ramírez-Romero et al. (2013) y García-Zamora et al. (2015).

En México se ha estimado que se generan 14.4 millones de $\mathrm{m}^{3} /$ mes de nejayote que, al descargarse en diferentes cuerpos de agua, aumentan la turbidez y disminuyen el oxígeno disuelto afectando la supervivencia de diferentes organismos acuáticos (RojasGarcía et al. 2012, Valderrama-Bravo et al. 2012, Alvarado-Granados et al. 2013, Ramírez-Romero et al. 2013, Ayala-Soto et al. 2014).

Ante tal problemática, se han propuesto modificaciones al proceso de nixtamalización tradicional para reducir la producción de este residuo y el consumo de agua (Janve et al. 2013, Méndez-Albores et al. 2014). Ferreira-Rolón et al. (2014) propusieron un tratamiento de lecho de lodos de flujo ascendente (UASB), alimentado de nejayote diluido con agua residual municipal, en el que obtuvieron una eficiencia de remoción de la DQO hasta del 90 \%. Dicho tratamiento permitió que el desecho cumpliera con las especificaciones de la norma oficial NOM-002-SEMARNAT-1996 (SEMARNAT 1996a) y con ello, poder ser descargado a los sistemas de alcantarillado urbano o municipal. Asimismo, Suárez-Meraz et al. (2016) propusieron un tratamiento de coagulaciónfloculación del nejayote, con el que lograron remover el $80 \%$ de la turbidez y cumplir con las especificaciones de la NOM-002-SEMARNAT-1996(SEMARNAT 1996a). Por otra parte, también se han planteado alternativas como utilizar el filtrado o el centrifugado con diferentes fines. Uno de ellos ha sido emplear el filtrado como medio de cultivo para la producción de probióticos y bacteriocinas (Salmerón-Alcocer et al. 
2003, Gutiérrez-Uribe et al. 2010, Ramírez-Romero et al. 2013). Otra aplicación ha sido la recuperación de carbohidratos por ultrafiltración para uso industrial (Valderrama-Bravo et al. 2012, Castro-Muñoz et al. 2015); sin embargo, el costo de la implementación de estos procesos es elevado (Durán-de Bazúa et al. 2007).

El uso de biopolímeros como el alginato de sodio (en adelante alginato) y el quitosano ha resultado ser una alternativa efectiva en la reducción de contaminantes en diferentes tipos de aguas residuales, como se describe posteriormente. El alginato es un polisacárido que está compuesto por residuos de D-manuronato (M) unidos por enlaces $\beta$ (1-4) y por monómeros de L-guluronato $(\mathrm{G})$ unidos por enlaces $\alpha$ (1-4). Además, las moléculas de $\mathrm{G}$ en este biopolímero otorgan la capacidad de formar un gel en presencia de iones divalentes como $\mathrm{Ba}^{+2}, \mathrm{Ca}^{+2} \mathrm{y}$ $\mathrm{Mg}^{+2}$ (Donati et al. 2005, Lozano-Álvarez et al. 2009, Lin et al. 2012). Diferentes grupos de investigación reportaron que el alginato puro o funcionalizado disminuye la carga orgánica de vinazas tequileras y remueve colorantes y iones metálicos (Blackburn 2004, Jáuregui-Rincón et al. 2011, Retes-Pruneda et al. 2014, Lozano-Álvarez et al. 2015, Ge et al. 2017, Skwarek et al. 2017, Tao et al. 2017, Yan et al. 2017). Su aplicación ha ido en aumento debido a que es biodegradable y de carácter inocuo para los seres vivos (Bolto y Gregory 2007, Donati y Paoletti 2009, Lin et al. 2012).

El quitosano resulta de la desacetilación de la quitina y está constituido principalmente por unidades de glucosamina unidas por enlaces $\beta$ (1-4) (Suárez-Meraz et al. 2016). Sus propiedades físicas y químicas como el carácter ácido-base, la solubilidad y la carga positiva, están relacionadas con los grupos amino presentes en su estructura, permitiendo la formación de enlaces con iones metálicos en soluciones cercanas a la neutralidad y con sustancias aniónicas en condiciones ácidas (Guibal y Roussy 2007, Quin et al. 2007). El quitosano y sus derivados han sido ampliamente utilizados para la remoción de materia orgánica, colorantes, iones metálicos y productos farmacéuticos (Cheng et al. 2005, Roussy et al. 2005, Guibal et al. 2006, Renault et al. 2009, Perju y Dragan 2010, Caldera et al. 2011, Jia et al. 2016, Saleha et al. 2017, Skwarek et al. 2017, Zhuo et al. 2017). Debido a la gran eficiencia que tienen los polisacáridos alginato y quitosano como agentes de remoción de contaminantes, en este trabajo se reporta su uso en la depuración del nejayote de manera separada y secuencial con el objeto de definir cuál método es el más eficiente.

\section{MATERIALES Y MÉTODOS}

\section{Obtención de la muestra de nejayote}

El nejayote se obtuvo de la nixtamalización de maíz blanco procedente de la región de Tamaulipas. Dicho proceso se realizó utilizando el método tradicional modificado de Salazar et al. (2014), el cual consistió en suspender $1 \mathrm{~kg}$ de maíz con $2 \mathrm{~L}$ de disolución de cal apagada al $1 \%(\mathrm{p} / \mathrm{v})$. El cocimiento se realizó a $97{ }^{\circ} \mathrm{C}$ durante $90 \mathrm{~min}$ (manteniendo el volumen total constante durante la cocción). Posteriormente, se dejó en reposo hasta alcanzar la temperatura de $28^{\circ} \mathrm{C}$, se separó el maíz (nixtamal) del agua de remojo (nejayote) y se realizaron dos lavados con agua purificada usando el mismo volumen del agua de cocción, dicho enjuague sirvió para desprender la cal y el pericarpio adheridos al maíz nixtamalizado. Este procedimiento produjo $18 \mathrm{~L}$ de nejayote por cada $3 \mathrm{~kg}$ de maíz. El agua de enjuague y el nejayote se mezclaron y se conservaron a $4{ }^{\circ} \mathrm{C}$ en ausencia de luz.

\section{Caracterización del nejayote}

Tres muestras de nejayote obtenidas de manera independiente se caracterizaron considerando los siguientes parámetros: DQO (mediante el uso de un digestor y espectrofotómetro marca Hach modelo DR 2700 que se apega a la norma NMXAA-030-SCFI-2012 (SCFI 2012); $\mathrm{DBO}_{5}$ según la norma NMX-AA-028-SCFI-2001 (SCFI 2001); pH; dureza según la norma NMX-AA-072-SCFI-2001 (SCFI (2001b); calcio con el método 2111-APHA (APHA, AWWA, WEF 1998); sólidos suspendidos totales (SST) y sólidos totales (ST) con el método 2540-APHA (APHA, AWWA, WEF 1998); sólidos sedimentables (SSED) según la norma NMX-AA004-SCFI-2013 (SCFI 2013); proteína (Bradford 1976); fibra cruda según la norma NMX-F-090-1978 (SEPAFIN 1978); fenoles totales según la norma NMX-AA-050-SCFI-2001 (CSFI 2001a) y azúcares reductores (Miller 1959). Se reportó el promedio obtenido de cada parámetro y su desviación estándar.

\section{Tratamiento del nejayote con alginato}

Con el fin de secuestrar los iones divalentes $\left(\mathrm{Ca}^{+2}\right.$

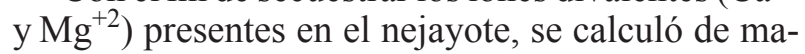
nera estequiométrica la cantidad de citrato de sodio para secuestrar dichos iones $\left(1073 \mathrm{mg} / \mathrm{L} \mathrm{de} \mathrm{Ca}^{2+}\right)$, de acuerdo con este cálculo se añadieron $2.6 \mathrm{~g} / \mathrm{L}$ de citrato de sodio. A $50 \mathrm{~mL}$ de esta mezcla se añadieron alícuotas de una solución acuosa de alginato (1, 2 y $3 \% \mathrm{p} / \mathrm{v}$ ). Después de mezclar los componentes, el alginato fue gelificado por la adición de $10 \mathrm{~mL}$ de $\mathrm{CaCl}_{2}$ al $5 \%(\mathrm{p} / \mathrm{v})$. Se dejó reposar la solución a $28^{\circ} \mathrm{C}$ 
en un baño de agua durante $24 \mathrm{~h}$. Finalmente, se separó el gel por filtración (usando papel Whatman No. 1 Maidstone, Inglaterra) y al sobrenadante se le determinaron las variables de respuesta. Para verificar la efectividad del polímero se utilizó un testigo (nejayote únicamente filtrado). Las variables de respuesta cuantificadas en todos los tratamientos fueron: el porcentaje de remoción de DQO, fenoles totales, azúcares reductores y ST. Todos los experimentos se realizaron por triplicado. El diseño experimental fue de tipo factorial (dos factores con tres niveles), los factores fueron la concentración de alginato $(0.16$, 0.33 y $0.50 \% \mathrm{p} / \mathrm{v})$ y el $\mathrm{pH}(4,8$ y 12$)$. Las variables de respuesta fueron DQO, fenoles, azúcares reductores y ST. A los datos se les hizo un análisis de varianza y para comprobar la diferencia entre los tratamientos se realizó una prueba de Tukey.

\section{Tratamiento del nejayote con quitosano soluble}

Se realizó un análisis de varianza de un factor para determinar la diferencia entre los tratamientos en los que se modificó la concentración del quitosano para evaluar su efectividad en la remoción de contaminantes del nejayote. A muestras de $50 \mathrm{~mL}$ de nejayote se les añadió $\mathrm{HCl}$ al $10 \%(\mathrm{v} / \mathrm{v})$ para ajustar el $\mathrm{pH}$ a un valor de 2, posteriormente se les añadió la cantidad necesaria de quitosano en polvo para obtener las concentraciones a evaluar $(0.2$ a $1.0 \% \mathrm{p} / \mathrm{v}$ ). Para la disolución del quitosano en nejayote, las soluciones obtenidas se dejaron reposar por $12 \mathrm{~h}$ a $28^{\circ} \mathrm{C}$. Posteriormente, se reajustó el $\mathrm{pH}$ de la solución de nejayote-quitosano a 2. Para separar el quitosano y los componentes del nejayote adsorbidos a él, se elevó abruptamente el $\mathrm{pH}$ a un valor de 11 con $\mathrm{NaOH}$ al $30 \%(\mathrm{p} / \mathrm{v})$. Se dejó reposar durante 30 min a $28{ }^{\circ} \mathrm{C}$ en baño de agua y se separó por filtración usando papel Whatman No.1 (Maidstone, Inglaterra). Al filtrado y al nejayote testigo (nejayote filtrado) se le determinaron las variables de respuesta (DQO, fenoles, azúcares reductores y ST). Todos los experimentos se realizaron por triplicado y se realizó un análisis de varianza de una vía con los datos obtenidos.

\section{Tratamiento del nejayote con quitosano en polvo (insoluble)}

Para el tratamiento de adsorción con quitosano en polvo de peso molecular medio (Sigma-Aldrich, Steinhem, Alemania) se utilizó el mismo diseño experimental que con el alginato. Los factores fueron: la concentración de quitosano $(0.5,1.0$ y $1.5 \%(\mathrm{p} / \mathrm{v})$ y el $\mathrm{pH}(4,8$ y 12$)$. Las muestras de nejayote se ajustaron a los diferentes valores de $\mathrm{pH}$ utilizando $\mathrm{NaOH}$ y $\mathrm{HCl}$ diluidos. Posteriormente se añadió quitosano en polvo al nejayote para ajustar su concentración al $0.5,1.0$ y $1.5 \%(\mathrm{p} / \mathrm{v})$ y tras un día de incubación con agitación orbital a $28^{\circ} \mathrm{C}$ y $150 \mathrm{rpm}$, se separó el polímero del nejayote por medio de filtración rápida (Whatman No. 6 Maidstone, Inglaterra) y se cuantificaron las variables de respuesta a las muestras tratadas con el polímero y a un testigo de nejayote filtrado.

\section{Tratamiento secuencial}

Para determinar el efecto del orden en la aplicación de los biopolímeros en las variables de respuesta, se realizaron tratamientos con alginato y quitosano de manera secuencial, alternando el orden de los polímeros utilizados. Las secuencias utilizadas fueron las siguientes: a) aplicación de alginato al nejayote y el efluente obtenido se sometió al tratamiento con quitosano soluble (alginato-quitosano) y b) aplicación de quitosano soluble al nejayote seguido del tratamiento con alginato al efluente resultante (quitosano-alginato). Cada uno de los tratamientos se realizó por triplicado bajo las condiciones óptimas obtenidas para la aplicación de los polímeros de manera independiente.

\section{Análisis estadístico}

La diferencia entre los tratamientos: a) alginato, b) quitosano, c) quitosano-alginato y d) alginatoquitosano se obtuvo con un análisis de varianza de un factor. La diferencia de medias se realizó con una prueba de Tukey con una significancia de $\mathrm{p} \leq 0.05$. Los análisis se realizaron en el programa estadístico R (Core 2013). La medición de los parámetros se hizo por triplicado, los resultados se expresaron como la media \pm desviación estándar.

\section{RESULTADOS Y DISCUSIÓN}

\section{Caracterización del nejayote}

Desde el punto de vista social y económico, los derivados del maíz son importantes, sin embargo, el nejayote producido en el proceso de la nixtamalización es altamente contaminante (Paredes-López et al. 2009, Ferreira-Rolón et al. 2014). La composición del nejayote varía en función del tipo de maíz, cantidad por lote, agua utilizada, cantidad de cal, y tiempo de cocción y de reposo (Campechano-Carrera et al. 2012). En el cuadro I se muestra la caracterización del nejayote en estudio y se contrasta con valores reportados por otros autores. Como puede observarse, para el nejayote en estudio se obtuvieron valores elevados de $\mathrm{pH}(10.9 \pm 0.46)$ debido al 
CUADRO I. CARACTERIZACIÓN DE LA MUESTRA DE NEJAYOTE

\begin{tabular}{lccl}
\hline pH & Nejayote & Valores reportados & Referencia \\
\hline Alcalinidad total $\left(\mathrm{mg} / \mathrm{L} \mathrm{CaCO}_{3}\right)$ & $2600 \pm 265$ & $\begin{array}{l}11.49 \pm 0.49 \\
12 \pm 0.2\end{array}$ & $\begin{array}{l}\text { Valderrama-Bravo et al. (2012) } \\
\text { García-Depraect et al. (2017) }\end{array}$ \\
\hline Fenoles totales $(\mathrm{mg} / \mathrm{L})$ & $31.81 \pm 2.86$ & $577.4 \pm 5.9$ & García-Depraect et al. (2017) \\
\hline Fibra cruda (\%) & $20.21 \pm 0.862$ & 22.77 & $\begin{array}{l}\text { Rosentrater (2006) } \\
\text { Argun y Argun (2017) }\end{array}$ \\
\hline Proteína cruda (\%) & 45.3 & García-Depraect et al. (2017) \\
\hline Azúcares reductores (mg/L) & $149 \pm 13.04$ & $148.6 \pm 3.2$ & García-Depraect et al. (2017) \\
\hline Dureza (mg/L CaCO 3$)$ & $1826 \pm 25$ & $5768.67 \pm 0.46$ & Valderrama-Bravo et al. (2012) \\
\hline Cenizas (\%) & $0.65 \pm 0.16$ & $0.767 \pm 0.014$ & Valderrama-Bravo et al. (2012) \\
\hline CaTot (mg/L) & $1073 \pm 125$ & $1526.21 \pm 26.53$ & Valderrama-Bravo et al. (2012) \\
\hline DQO (mg/L) & $57900 \pm 624$ & $25080.0 \pm 563.0$ & García-Depraect et al. (2017) \\
\hline ST (mg/L) & $29006 \pm 839$ & $24466.7 \pm 638.9$ & García-Depraect et al. (2017) \\
\hline SST (mg/L)* & $18900 \pm 900$ & $8342 \pm 25.3$ & Valderrama-Bravo et al. (2012) \\
\hline SSED (mL/L)* & $410 \pm 10$ & $>100$ & Valderrama-Bravo et al. (2012) \\
\hline
\end{tabular}

* Valores que sobrepasan los niveles máximos permisibles establecidos por la norma oficial mexicana: NOM001-SEMARNAT-1996 y NOM-002-SEMARNAT-1996 (para descarga a ríos: $200 \mathrm{mg}$ /L de sólidos suspendidos totales (SST); $1 \mathrm{~mL} / \mathrm{L}$ de sólidos sedimentables (SSED), en uso de suelo para riego agrícola y descarga a drenaje urbano o municipal, ambos parámetros no aplican), $\mathrm{ST}=$ sólidos totales, quitosano = tratamiento con quitosano soluble al $0.8 \%(\mathrm{v} / \mathrm{v})$, alginato $=$ tratamiento con alginato de sodio al $0.33 \%(\mathrm{p} / \mathrm{v})$

exceso de $\mathrm{Ca}(\mathrm{OH})_{2}$, también se afectó el valor de la dureza $\left(1826 \pm 25 \mathrm{mg} / \mathrm{L} \mathrm{CaCO}_{3}\right)$, las cenizas $(0.65 \pm$ $0.16 \%)$, la alcalinidad total $(2600 \pm 265 \mathrm{mg} / \mathrm{L}$ $\left.\mathrm{CaCO}_{3}\right)$ y la concentración de CaTot $(1073 \pm 125$ $\mathrm{mg} / \mathrm{L}$ ). El proceso de cocción y lavado modifica los componentes del maíz, permite el desprendimiento del pericarpio y eleva la cantidad de SST (18 900 $\pm 900 \mathrm{mg} / \mathrm{L})$ y SSED $(410 \pm 10 \mathrm{~mL} / \mathrm{L})$, aunque no en la misma proporción, pero sí en cantidades elevadas. Valderrama-Bravo et al. (2012) reportaron $8342 \pm 25.3 \mathrm{mg} / \mathrm{L}$ de SST y una cantidad $>100$ $\mathrm{mL} / \mathrm{L}$ de SSED. La variedad del grano utilizado y las diferencias entre los procesos de nixtamalización empleados en cada estudio generan una variación en los resultados (desviación estándar). Entre los principales componentes del nejayote se encuentran: antioxidantes (fenoles totales $31.81 \pm 2.86 \mathrm{mg} / \mathrm{L}$ ), hemicelulosa y celulosa (fibra cruda $=20.21 \pm$ $0.862 \%$ ), azúcares reductores del endospermo (149 $\pm 13.04 \mathrm{mg} / \mathrm{L}$ ), proteínas (proteína cruda $=6.125 \pm$ $0.671 \%$ ), así como carotenoides que le dan el color amarillo característico a este efluente. La presencia de estos componentes en solución o suspensión ocasiona que el nejayote sea altamente contaminante (Berlanga-Reyes et al. 2011, Valderrama-Bravo et al. 2012). Diferentes autores, como Suárez-Meraz et al. (2016); Castro-Muñoz et al. (2015); FerreiraRolón et al. (2014) y Valderrama-Bravo et al. (2012) caracterizaron el nejayote y reportaron valores de $\mathrm{pH}$ entre 11.39 y 13.4 , intervalos de DQO de 24600 a $40058 \mathrm{mg} \mathrm{O}_{2} / \mathrm{L}$, concentraciones de $\mathrm{Ca}_{\mathrm{Tot}}$ de 955.7 a $1526 \mathrm{mg} / \mathrm{L}$ y dureza de 5768 a $7500 \mathrm{mg} / \mathrm{L}$. La concentración de $\mathrm{Ca}_{\mathrm{Tot}}$ encontrada en la muestra de nejayote tratada en este estudio se encuentra en el intervalo mencionado por dichos autores. Por otro lado, el valor de $\mathrm{pH}$ encontrado se encuentra muy cerca del límite inferior reportado $(11.49 \pm 0.49$ a $12 \pm 0.2)$ (Valderrama-Bravo et al. 2012, García-Depraect et al. 2017). Sin embargo, los valores de la DQO y dureza están fuera del rango encontrado por estos autores. Las diferencias en el proceso, como el tipo de agua, tiempos de reposo y el tipo de maíz empleado en la nixtamalización se ven reflejados en la variabilidad de los parámetros analizados. 
El proceso de nixtamalización, como ya se ha mencionado, provoca el desprendimiento de pericarpio, germen y endospermo, lo que aumenta el material suspendido y sedimentable, debido a esto los SST y SSED se elevan (Suárez-Meraz et al. 2016). La muestra de nejayote rebasó los límites máximos establecidos por las normas oficiales mexicanas NOM-001-SEMARNAT-199, NOM002SEMARNAT-1996 y SEMARNAT 1996, 1996a (ver Cuadro I). Otros autores encontraron valores similares a los obtenidos en la muestra de estudio, por ejemplo, Pulido et al. (1987) reportaron una concentración de SST de 20000 mg/L y Durán-de Bazúa et al. (2007) encontraron una concentración de $2400 \mathrm{mg} / \mathrm{L}$ de SST.

Por otro lado, aunque no se ha establecido el límite máximo permisible para la DQO de una descarga, diferentes grupos de investigación coinciden en que los valores de la DQO reportados para el nejayote son elevados (10 200 a 22200 mg/L) (Durán-de Buzúa et al. 2007, García-Zamora et al. 2015, Suárez-Meraz et al. 2016). A pesar de que la DQO no se considera en la NOM-002-SEMARNAT-1996 (SEMARNAT 1996a), su determinación es importante en la caracterización del nejayote y en la evaluación de la eficiencia de los tratamientos aplicados en este trabajo, debido a que este parámetro permite conocer la cantidad total de materia orgánica que se quiere disminuir con los tratamientos propuestos.

\section{Tratamiento del nejayote con alginato}

Con el objetivo de disminuir la carga orgánica del nejayote se estudió la influencia del $\mathrm{pH}$ y la concentración de alginato en la disminución de la DQO, concentración de fenoles, azúcares reductores y ST de este residuo. La prueba de análisis de varianza muestra que la concentración de alginato y el $\mathrm{pH}$, así como las interacciones de estos factores influyen de forma significativa ( $p$ $<0.05)$ en la remoción de los contaminantes del nejayote tales como la DQO, fenoles, azúcares y ST (Cuadro II). La mejor combinación para remover la DQO es a pH 4 con una concentración de alginato al $0.33 \%$. En la figura 1a se observa que se logra una remoción de $61.2 \%$.

La utilización de concentraciones menores a este valor implica también una menor concentración de sitios de interacción molecular, lo que se refleja en un bajo nivel de remoción de la DQO. Por otro lado, el incremento en la concentración de alginato $(0.5 \%)$ disminuyó también los valores de remoción de la DQO, debido a que el exceso de dicho polímero provoca su estabilización (Bolto y Gregory 2007). Se observó que valores de $\mathrm{pH} 4$ permitieron una mayor remoción de contaminantes. Lo anterior puede ser debido a que a un $\mathrm{pH} 4$ existe una mayor proporción de moléculas de alginato que están desprotonadas, pues los valores de $\mathrm{pKa}$ de los grupos carboxilo del G y M son de 3.65 y 3.38, respectivamente (Maurstad et al. 2003). Esto permite que el alginato interaccione con $\mathrm{el} \mathrm{Ca}^{+2} \mathrm{y}$ forme un gel muy estable de alginato de calcio, de fácil manejo para separarlo del sobrenadante.

Se ha reportado que los valores de $\mathrm{pH}$ ácidos favorecen el establecimiento de interacciones hidrofóbicas y puentes de hidrógeno entre el biopolímero y los diferentes componentes solubles e insolubles de la muestra (Berlanga-Reyes et al. 2011, ValderramaBravo et al. 2012, Ramírez-Romero et al. 2013). Cuando el gel de alginato de calcio se forma en presencia del nejayote, una fracción de los sólidos disueltos y suspendidos quedan adsorbidos y atrapados en los espacios presentes entre los dímeros del alginato. La formación de dichos espacios se origina por la acción cohesiva del ion de calcio al interactuar con los grupos carboxilo, hidroxilo y con los átomos de oxígeno que forman el enlace glucosídico y el enlace hemiacetálico de los residuos de $\mathrm{G}$ de la cadena polimérica (Lin et al. 2012).

Con respecto a los fenoles (Fig. 1b), se observó una variación del porcentaje de remoción de estos compuestos en función del $\mathrm{pH}$. A pesar de la repulsión inicial ejercida por los grupos carboxilo ionizados presentes en los residuos de $\mathrm{M}$ y $\mathrm{G}$, se obtuvo la mayor remoción a $\mathrm{pH}=4(75 \%)$. Por otro lado, la menor remoción fue de $63 \%$ y se obtuvo a pH 8.

En el caso de la remoción de los azúcares (Fig. 1c), se observó que al incrementarse el pH la eficiencia de remoción también aumentó, lo cual sugiere que las fuerzas de cohesión entre los grupos carboxilo del alginato y los azúcares del nejayote son lo suficientemente fuertes para aumentar su remoción, como ya lo han reportado Meng et al. (2015). La eficiencia de remoción máxima de azúcares por el alginato fue del $94.5 \%$ cuando el $\mathrm{pH}$ se mantuvo fijo (4, 8 y 12$)$. De hecho, no se observaron diferencias significativas en la remoción de azúcares en los tres valores de $\mathrm{pH}$, salvo a pH 4 y concentración de alginato de $0.33 \%$, donde una ligera turbidez de la solución subestimó la eficiencia de remoción observada.

La característica física más importante de las aguas residuales es el contenido de ST, los cuales comprenden la materia en suspensión, sedimentable y disuelta (Sahu y Chaudhari 2013). De manera general se obtuvieron valores bajos de remoción debido a la presencia de iones $\mathrm{Ca}^{+2}, \mathrm{Cl}^{-1}$, citrato, $\mathrm{Na}^{+1}$, etc., 
CUADRO II. PRUEBA DE ANÁLISIS DE VARIANZA DE DOS FACTORES (ALGINATO Y pH) PARA EL TRATAMIENTO CON ALGINATO SOLUBLE

\begin{tabular}{lrrlll}
\hline \multicolumn{5}{c}{ a) Remoción de DQO } \\
\hline Fuente & GL & \multicolumn{1}{c}{ SC } & CM & p \\
\hline Alginato (\%) & 3 & 6499221111 & 2166407037 & 0.000 & \\
pH & 2 & 60723889 & 30361944 & 0.000 & \\
Interacción & 6 & 43927222 & 7321204 & 0.000 & \\
Error & 24 & 11546667 & 481111 & & \\
Total & 35 & 6615418889 & & & \\
\hline
\end{tabular}

R-cuad. $=99.83 \%$

Modelo lineal general. DQO vs. alginato, $\mathrm{pH}$

DQO = 34694.4 + 23205.6 A0 - 6838.9 A0.16 - 9394.4 A0.33 + 522.2 pH12 - 1786.1 pH4 + 1786.1 A0*pH4 - 655.6 A0.33*pH12 - 1047.2 A0.33*pH4

\begin{tabular}{lrrrr}
\hline \multicolumn{5}{c}{ b) Remoción de fenoles } \\
\hline Fuente & $\mathrm{GL}$ & $\mathrm{SC}$ & $\mathrm{CM}$ & $\mathrm{p}$ \\
\hline Alginato (\%) & 3 & 3099.16 & 1033.05 & 0.000 \\
$\mathrm{pH}$ & 2 & 1406.82 & 703.41 & 0.000 \\
Interacción & 6 & 514.24 & 85.71 & 0.000 \\
Error & 24 & 53.86 & 2.24 & \\
Total & 35 & 5074.08 & & \\
\hline
\end{tabular}

R-cuad. $=98.94 \%$

Modelo lineal general. Fenoles vs. alginato, pH

Fenoles $=\mathbf{2 4 . 2 5 9 9}+15.0339$ A0 -4.0715 A0.16 -0.9169 A0.33 -3.8566 pH12 -9610 pH4 + 3.8566 A0*pH12 + 4.9610 A0*pH4 - 2.0880 A0.16*pH12 - 2.8942 A0.33*pH12 - 1.5493 A0.33*pH4

\begin{tabular}{lrccc}
\hline \multicolumn{5}{c}{ c) Remoción de azúcares } \\
\hline Fuente & $\mathrm{GL}$ & $\mathrm{SC}$ & $\mathrm{CM}$ & $\mathrm{p}$ \\
\hline Alginato (\%) & 3 & 0.073050 & 0.0243501 & 0.000 \\
$\mathrm{pH}$ & 2 & 0.017005 & 0.0085024 & 0.000 \\
Interacción & 6 & 0.016691 & 0.0027819 & 0.000 \\
Error & 24 & 0.007680 & 0.0003200 & \\
Total & 35 & 0.114427 & & \\
\hline
\end{tabular}

R-cuad. $=93.29 \%$

Modelo lineal general. Azúcares vs. alginato, $\mathbf{p H}$

azúcares $=\mathbf{0 . 1 0 3 9 0 9}+0.071791 \mathbf{A 0}-0.017866 \mathbf{A 0 . 1 6}-0.030611 \mathbf{p H 1 2}+0.017707 \mathbf{p H 4}+0.030611$ A0*pH12 - 0.017708 A0*pH4 - 0.030458 A0.16*pH4 - 0.019068 A0.33*pH12 + 0.037025 A0.33*pH 4

d) Remoción de ST

\begin{tabular}{lrrrr}
\hline Fuente & GL & \multicolumn{1}{c}{ SC } & \multicolumn{1}{c}{ CM } & \multicolumn{1}{c}{ p } \\
\hline Alginato (\%) & 3 & 81838149 & 27279383 & 0.000 \\
pH & 2 & 819758 & 409879 & 0.000 \\
Interacción & 6 & 3355925 & 559321 & 0.000 \\
Error & 24 & 823964 & 34332 & \\
Total & 35 & 86837796 & & \\
\hline
\end{tabular}

R-cuad. $=99.05 \%$

Modelo lineal general. ST vs. alginato, $\mathrm{Ph}$

$\mathbf{S T}=\mathbf{2 6 5 1 0 . 1}+2509.89 \mathbf{A 0}-172.89$ A0.16 - 1040.22 A0.33 + 130.72 pH4 - 386.28 A0.16*pH12 + 459.39 A0.33*pH12 + 226.06 A0.33*pH4

$\mathrm{DQO}=$ demanda química de oxígeno, $\mathrm{ST}=$ sólidos totales, $\mathrm{GL}=$ grados de libertad, $\mathrm{SC}=$ suma de cuadrados, $\mathrm{CM}=$ cuadrado medio, $\mathrm{p}=$ probabilidad 

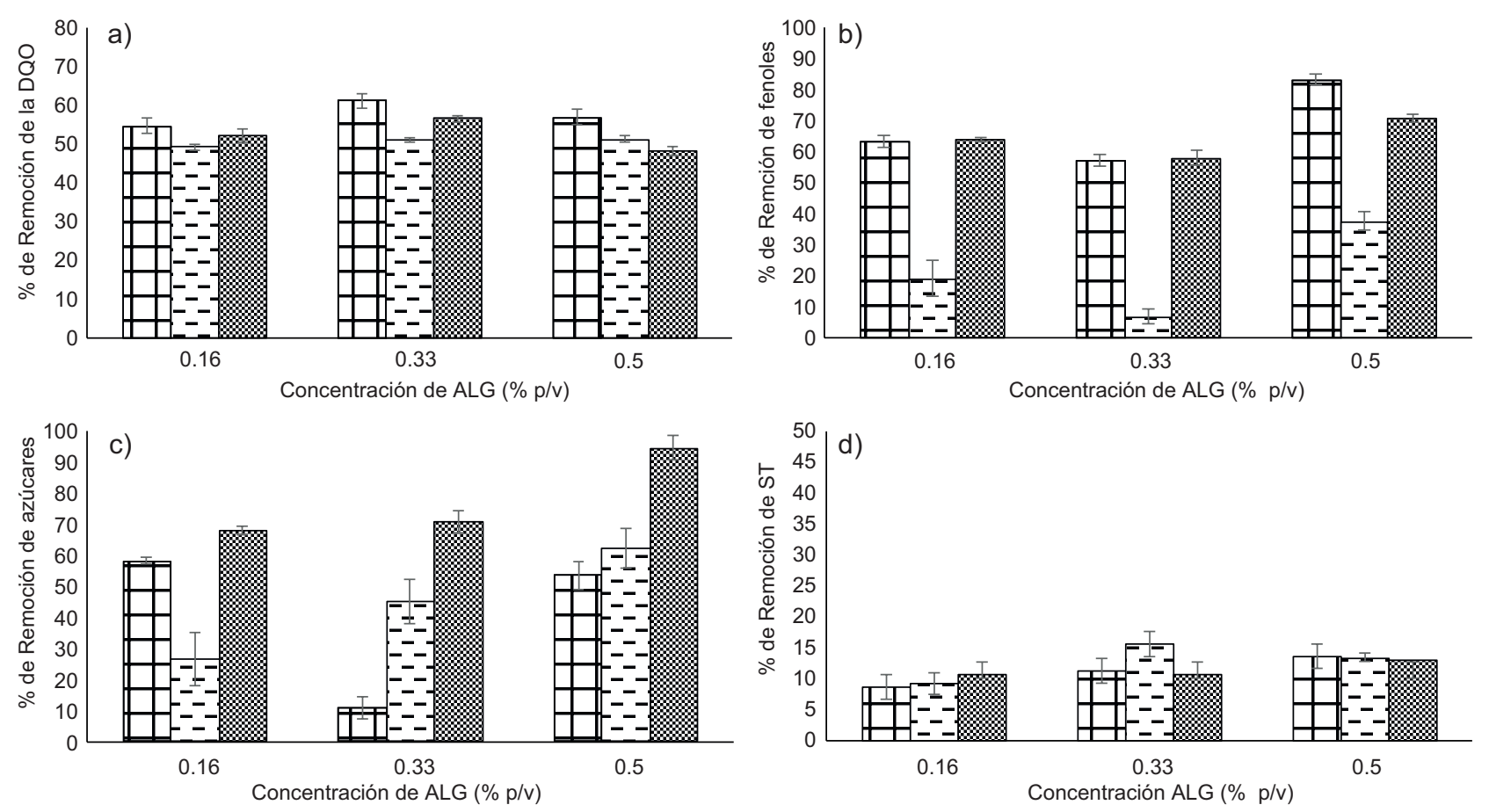

$\square \mathrm{pH} 4 \quad \square \mathrm{pH} 8 \quad \mathrm{QpH} 12$

Fig. 1. Porcentaje de remoción de contaminantes en función de la concentración de alginato $(0.16,0.33$ y $0.50 \%$ (p/v)) y el pH (4, 8 y 12): a) demanda química de oxígeno (DQO), b) fenoles, c) azúcares y d) sólidos totales ( $\mathrm{ST} ; \mathrm{p} \leq 0.05)$

en el filtrado, que evita obtener valores reales de la remoción. Para visualizar esta situación se aplicó la metodología propuesta a una muestra de agua destilada usada como testigo y se obtuvo un rango de ST de 6125 a $6537 \mathrm{mg} / \mathrm{L}$ independientemente del pH y la concentración de alginato. Se observa que al incrementar la concentración de alginato se obtienen valores de remoción de ST mayores. Sin embargo, se encontró que el máximo de remoción fue del 15 $\%$ a pH 8 con $0.33 \%$ (p/v) de alginato (Fig. 1d). Lo anterior puede deberse a varios efectos involucrados en la remoción de ST. Primero, a medida que el $\mathrm{pH}$ se incrementa, la repulsión entre el alginato, las moléculas y las partículas presentes en el nejayote es mayor, por lo que un $\mathrm{pH}$ intermedio favorece una mayor remoción. Segundo, a pH 8 la concentración intermedia del alginato presenta una mayor remoción de ST, lo cual sugiere un comportamiento típico de un agente floculante como el alginato, donde concentraciones bajas del polímero dificultan una buena floculación y las concentraciones altas sobre estabilizan a las partículas que están contenidas en el nejayote (Singh et al. 2000, Sahu y Chaudhari 2013). Por último, es importante mencionar que la cantidad adicionada de citrato de sodio que actuó como agente secuestrante del $\mathrm{Ca}^{2+}$, se disolvió completamente en el nejayote. Lo anterior provocó que al realizar la gelificación y la filtración los iones solubles (citrato, $\mathrm{Na}^{+} \mathrm{y} \mathrm{Ca}^{2+}$ ) terminaran en la fase acuosa, produciendo un valor alto de ST. Esto resultó en una baja eficiencia de remoción de los mismos.

\section{Tratamiento con quitosano soluble}

En este estudio se analizó la capacidad de remoción con base en los principios de adsorciónprecipitación del quitosano, a diferencia del proceso de coagulación-floculación reportado por Guibal y Roussy (2007) y Suárez-Meraz et al. (2016). De esta manera, el quitosano soluble adsorbe a los sólidos suspendidos y disueltos presentes en el nejayote y posteriormente una elevación abrupta en el $\mathrm{pH}$ precipita al producto quitosano-nejayote. En la figura 2 se muestra el porcentaje de remoción de la DQO, fenoles totales, azúcares reductores y ST en función de la concentración de quitosano soluble. El tratamiento se llevó a cabo a $\mathrm{pH}$ ácido $(\mathrm{pH}=2)$ para lograr solubilizar el quitosano en el nejayote, luego, se incrementó drásticamente el $\mathrm{pH}$ (hasta $\mathrm{pH}=11$ ) para insolubilizar al polímero con los contaminantes unidos a él. El mayor porcentaje de remoción de la 

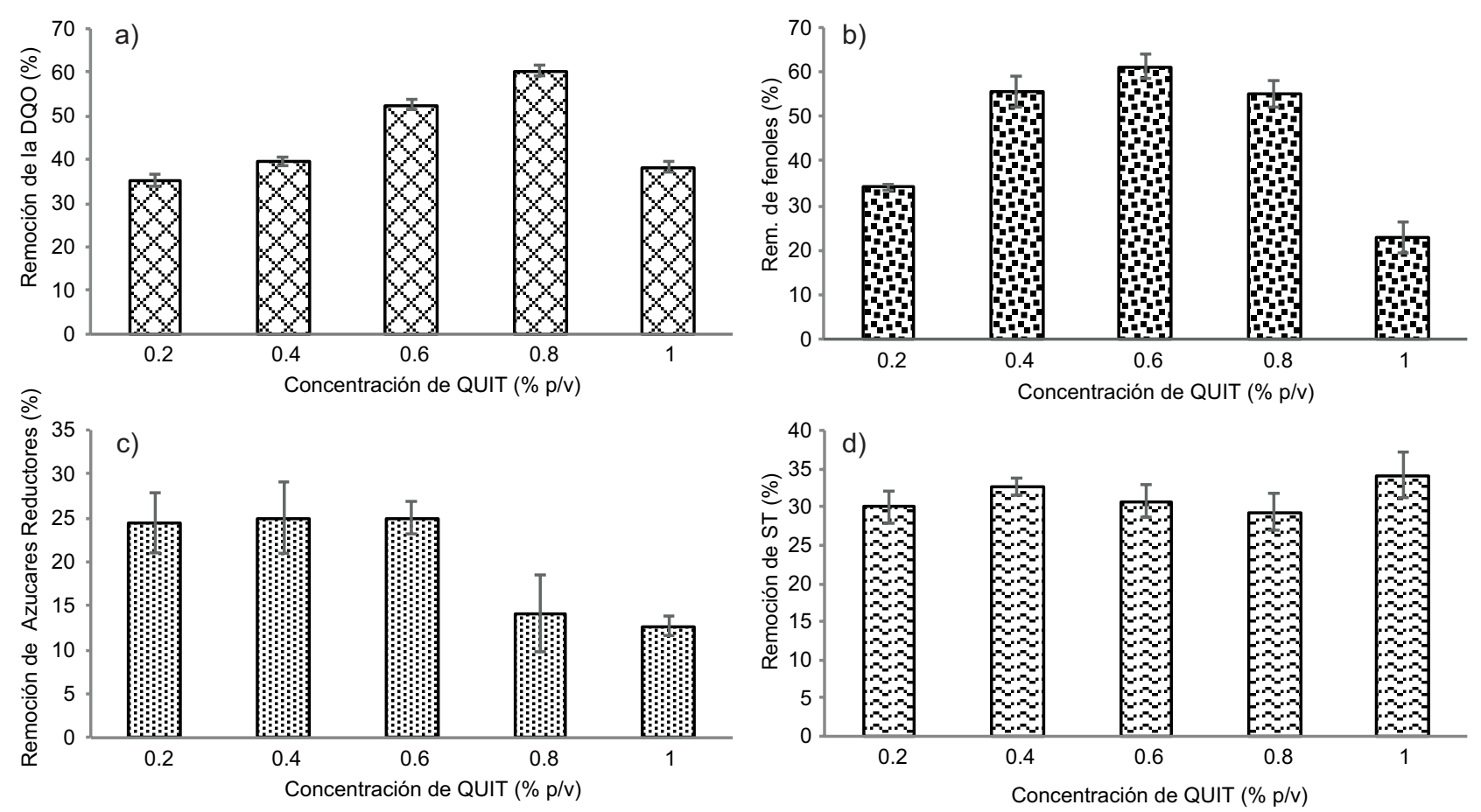

Fig. 2. Porcentaje de remoción de contaminantes en función de la concentración de quitosano soluble: $0.2,0.4,0.6,0.8$ y $1 \%$ (p/v). a) demanda química de oxígeno (DQO), b) fenoles, c) azúcares y d) sólidos totales (ST; p $\leq 0.05$ )

DQO fue del $60 \%$ cuando se utilizó quitosano al $0.8 \%$ $(\mathrm{p} / \mathrm{v})$. La mayor remoción de fenoles fue del $61 \%$ con una concentración de $0.6 \%$ (p/v) de quitosano. El análisis de varianza (Cuadro III) mostró diferencia significativa entre los tratamientos de nejayote a diferentes concentraciones de quitosano $(\mathrm{p}<0.05)$. Éste posee diferentes mecanismos por los cuales interactúa con el material presente en solución acuosa, los cuales se describen a continuación. Al poseer una carga positiva debido a los grupos amino protonados en soluciones con valores de $\mathrm{pH}$ ácido, neutraliza la carga de partículas coloidales con carga negativa y promueve la formación de grandes agregados (coloide-quitosano), debido a que las moléculas de este polímero actúan como agentes entrelazantes entre las partículas coloidales promoviendo la agregación y la sedimentación del material coloidal suspendido. Adicionalmente, las moléculas de quitosano pueden adsorber material disuelto presente en la fase acuosa. Posteriormente, el material disuelto y el coloidal, adsorbidos al quitosano, se pueden remover por sedimentación o filtración. Renault et al. (2009) y Guibal y Roussy (2007) han descrito que la remoción de diferentes materiales presentes en medio acuoso se puede representar por una gráfica, en forma de campana, de eficiencia de remoción en función de la dosis de quitosano. Este mismo comportamiento se obtuvo en el tratamiento de nejayote. Cuando se añadieron concentraciones bajas, el material coloidal suspendido y disuelto fue parcialmente neutralizado y adsorbido a este polímero, siendo insuficiente para lograr la sedimentación de todo el material presente en la fase acuosa. Por esta razón, la eficiencia de remoción es baja a dosis pequeñas de quitosano. Cuando se llega a la cantidad óptima de añadida, se dice que se llega al máximo de la campana y que todo el material coloidal y disuelto se encuentra unido a este biopolímero y, en consecuencia, su separación es eficiente mediante sedimentación o filtración. Sin embargo, si la cantidad de polímero está en exceso, el $\mathrm{pH}$ ácido de la solución lo hidroliza por $\beta$-eliminación con una magnitud que es proporcional a la concentración de quitosano, de tal manera que se generan mono y oligosacáridos que no precipitan al elevar el $\mathrm{pH}$, consecuentemente la eficiencia de remoción se ve disminuida. Es importante mencionar que la naturaleza y concentración de los contaminantes contenidos en el medio acuoso determinarán la cantidad que se añadirá de quitosano para removerlos de la solución acuosa. Guibal y Roussy (2007) encontraron que para remover colorantes la cantidad óptima de es de $43 \mathrm{mg} / \mathrm{L}$, Pinotti et al. (1997) reportaron que hasta $100 \mathrm{mg} / \mathrm{L}$ de este polímero se requieren para 
CUADRO III. ANÁLISIS DE VARIANZA UNIDIRECCIONAL: DQO VS. QUITOSANO PARA TRATAMIENTO DE NEJAYOTE CON QUITOSANO SOLUBLE

\begin{tabular}{lrrrr}
\hline \multicolumn{5}{c}{ a) Remoción de DQO } \\
\hline Fuente & GL & \multicolumn{1}{c}{ SC } & CM & \multicolumn{1}{c}{ p } \\
\hline Quitosano (\%) & 5 & 2271125000 & 454225000 & 0.000 \\
Error & 12 & 7220000 & 601667 & \\
Total & 17 & 2278345000 & & \\
\hline
\end{tabular}

R-cuad. $=99.68 \%$

Modelo lineal general. DQO vs. quitosano (\%)

DQO = 34716.7 + 23183.3 Q0 -4416.7 Q0.2 -7483.3 Q0.6 -11983.3 Q0.8 + 1190.5 Q1

\begin{tabular}{lrrrr}
\hline \multicolumn{5}{c}{ b) Remoción de fenoles } \\
\hline Fuente & GL & \multicolumn{1}{c}{ SC } & CM & p \\
\hline Quitosano (\%) & 5 & 183.961 & 36.792 & 0.000 \\
Error & 12 & 36.541 & 3.045 & \\
Total & 17 & 220.502 & & \\
\hline
\end{tabular}

R-cuad. $=83.43 \%$

Modelo lineal general. DQO vs. quitosano (\%)

Fenoles $=26.3139+5.4994$ Q0 -4.7472 Q0.6 $+1.0028 \mathbf{Q 0 . 8}+1.7871$ Q1

\begin{tabular}{lrccc}
\hline \multicolumn{5}{c}{ c) Remoción de azúcares } \\
\hline Fuente & GL & SC & CM & p \\
\hline Quitosano (\%) & 5 & 0.071921 & 0.014384 & 0.000 \\
Error & 12 & 0.006213 & 0.000518 & \\
Total & 17 & 0.078134 & & \\
\hline \multicolumn{5}{c}{ R-cuad. $=93.63 \%$}
\end{tabular}

Modelo lineal general. Azúcares vs. quitosano (\%)

Azúcares $=\mathbf{0 . 1 8 0 1 1 2}+0.11090$ Q0 -0.05078 Q0.4 - 0.06711 Q0.6 -0.04940 Q0.8 +0.04790 Q1

\begin{tabular}{lrrrr}
\hline \multicolumn{5}{c}{ d) Remoción de ST } \\
\hline Fuente & GL & SC & CM & p \\
\hline Quitosano (\%) & 5 & 137195178 & 27439036 & 0.000 \\
Error & 12 & 13600 & 1133 & \\
Total & 17 & 137208778 & & \\
\hline
\end{tabular}

R-cuad. $=99.99 \%$

Modelo lineal general: ST vs. quitosano (\%)

ST = 17301.1 + 6112.22 Q0- 891.11 Q0.2 -1524.44 Q0.4 -1081.11 Q0.6 - 731.11 Q0.8 - 1814.29 Q1

$\mathrm{DQO}=$ demanda química de oxígeno

remover residuos en forma de emulsión de la industria de alimentos, Guerrero et al. (1998) utilizaron quitosano $(100 \mathrm{mg} / \mathrm{L})$ para remover sólidos suspendidos de la industria pesquera, Li et al. (2013) necesitaron de $20 \mathrm{mg} / \mathrm{L}$ para remover a la bentonita de suspensiones acuosas y Suárez-Meraz et al. (2016) reportaron que la cantidad óptima para remover material coloidal de nejayote fue de $47 \mathrm{mg} / \mathrm{g}$ de nejayote sólido removido.
En el caso del nejayote sin tratar, se encontró que se requiere de $0.6 \%$ y $0.8 \%(\mathrm{p} / \mathrm{v})$ de quitosano para lograr una remoción máxima de la DQO y fenoles, respectivamente (Figs. 2a y 2b). Se puede observar que los datos mostrados en estas figuras tienen una tendencia de campana. Estos resultados sugieren que el material coloidal interacciona con el quitosano de manera similar a una coagulación-floculación en su fase inicial. Es 
decir, los sólidos suspendidos y disueltos se adsorben al quitosano, pero, al precipitarlos con el cambio abrupto de $\mathrm{pH}$ el comportamiento es diferente al observado en un proceso de floculación-sedimentación. En lo referente a los azúcares reductores se alcanzó el $25 \%$ de remoción a concentraciones de 0.2 a $0.6 \%(\mathrm{p} / \mathrm{v})$ de quitosano. En el caso de estos últimos (Fig. 2c), no se observa el mismo perfil de remoción que en los casos anteriores debido a que los azúcares reductores cuantificados en el nejayote después del tratamiento con quitosano son en su mayoría solubles y sólo una fracción de ellos se une a este polisacárido. En consecuencia, se observa que se requiere entre 0.2 y $0.6 \%$ para obtener una remoción de azúcares considerable ( $25 \%$ en promedio). Se observó que al incrementar la concentración del biopolímero la remoción de azúcares disminuye, lo que sugiere que hay una hidrólisis del quitosano que libera azúcares que son reductores, por lo tanto, la eficiencia de remoción se ve afectada.

Por otra parte, para los ST la eficiencia de remoción fue del 30 al $34 \%$. Estos resultados concuerdan con los de Suárez-Meraz et al. (2016) quienes reportan que las interacciones electrostáticas entre coloides del nejayote y el quitosano tienen un papel importante para la remoción de sólidos. La figura 2d muestra que el porcentaje de remoción, aunque existe diferencia significativa entre los tratamientos (Cuadro IIId), es poco dependiente de la cantidad de quitosano. El quitosano gelificado obtenido de cada tratamiento (quitosano-nejayote), se separó con una membrana de tipo cualitativo (Whatman No. 1 Maidstone, Inglaterra) permitiendo pasar sólidos menores a $3 \mu \mathrm{m}$. Cabe señalar que la formación del gel atrapa los sólidos sedimentables y suspendidos y parte del material coloidal. Al final se cuantificó la cantidad de ST como variable de respuesta y se obtuvo una remoción de 30.5 a $34.5 \%$ de ST, mientras que el nejayote testigo solamente removió un $13 \pm 0.5 \%$. Suárez-Meraz et al. (2016) han reportado resultados eficientes en la remoción de la turbiedad $(80 \%)$ del nejayote con quitosano. Sin embargo, a diferencia de ellos que utilizaron nejayote centrifugado, en este estudio el tratamiento se aplicó a muestras de nejayote completo. El proceso propuesto en este trabajo disminuye el equipo y operaciones unitarias empleadas para su tratamiento y, en consecuencia, su costo.

\section{Tratamiento con quitosano en polvo (insoluble)}

Para evaluar la eficiencia del quitosano en polvo, se midió la remoción de contaminantes en función de su concentración $(0.1,0.5,1$ y $1.5 \%$ p/v) y el pH (4, 8 y 12). Dichas concentraciones fueron utilizadas teniendo en cuenta que, a valores inferiores de concentración, las eficiencias de remoción estuvieron por debajo de aquellas obtenidas con quitosano soluble, ya que con este tratamiento se midió la capacidad de remoción por el fenómeno de adsorción. El análisis de varianza (Cuadro IV) muestra diferencia significativa entre los tratamientos $(p<0.05)$, además existen interacciones significativas entre el quitosano y el $\mathrm{pH}$, siendo la mejor combinación de $\mathrm{pH} 8$ y quitosano de 1.5. Esto se observa en la figura 3, donde al utilizar una concentración de quitosano al $0.1 \%$, se logró una remoción de la DQO entre 19 y $25 \%$ en el intervalo de $\mathrm{pH}$ utilizado. Del mismo modo se removió entre un 15 y $29 \%$ de fenoles y entre un 15 y $18 \%$ de azúcares. En la figura 3a se muestra el porcentaje de remoción de la DQO en función de la concentración de quitosano insoluble y el $\mathrm{pH}$. $\mathrm{Al}$ incrementar la concentración de quitosano no se afectó la eficiencia de remoción de la DQO en el intervalo de $\mathrm{pH} 8$-a 12. Sin embargo, a pH 8 y concentración de quitosano del $1.5 \%$, se observó un máximo en la DQO (48\%), lo que sugiere que el biopolímero está interaccionando de manera más eficiente con los materiales incluidos en el nejayote. El grado de desacetilación del quitosano (85\%), sugiere que a pH 8 , los grupos amino están parcialmente protonados, lo que les confiere una considerable carga positiva. Debido a que las moléculas y partículas presentes en el nejayote poseen carga negativa, se genera una atracción de tipo electrostático que incrementa el valor de eficiencia en la remoción de la DQO dada por los puentes de hidrógeno. Además existen interacciones hidrofóbicas que favorecen la disminución de la DQO a valores superiores de $\mathrm{pH}$ (Bolto y Gregory 2007). La figura 3a muestra que un incremento posterior a un $\mathrm{pH}$ de 12 desprotona completamente al quitosano y que la repulsión ejercida por sus moléculas hacia las moléculas y partículas presentes en el nejayote es lo suficientemente grande como para evitar un incremento importante en el porcentaje de remoción de la DQO. Por otro lado, se observó que a $\mathrm{pH} 4$ las eficiencias de remoción de la DQO disminuyen y llegan a un mínimo cuando la concentración de quitosano es de $1.5 \%$. Wang et al. (2006) encontraron que el $\mathrm{pKa}$ del quitosano se encuentra entre 6.17 y 6.51 , lo cual sugiere que los grupos amino de las unidades estructurales de este polímero (glucosamina), se protonan y adquieren una carga positiva a $\mathrm{pH} 2$. en consecuencia, la solubilidad de este polisacárido se ve incrementada. Lo anterior se traduce en un aumento en el valor de la DQO final y, como resultado, la eficiencia de remoción disminuye de manera drástica. 
CUADRO IV.PRUEBA DE ANÁLISIS DE VARIANZA DE DOS FACTORES (QUITOSANO, pH): PARA TRATAMIENTO DE NEJAYOTE CON QUITOSANO EN POLVO

\begin{tabular}{lrrrr}
\hline \multicolumn{5}{c}{ a) Remoción de DQO } \\
\hline Fuente & GL & SC & CM & p \\
\hline Quitosano (\%) & 3 & 458667249 & 64889226 & 0.000 \\
pH & 2 & 129778452 & 152889083 & 0.000 \\
Interacción & 6 & 231829717 & 38638286 & 0.000 \\
Error & 24 & 14613757 & 608907 & \\
Total & 35 & 834889173 & & \\
\hline
\end{tabular}

R-cuad. $=98.25 \%$

Modelo lineal general. DQO vs. quitosano en polvo, $\mathrm{pH}$

DQO = 34684.5 + 5208.9 Q0.1 -4329.0 Q0.5 -1973.4 Q1 - 1093.5 Q1.5 + 2219 pH4-2418.9 pH8 + 199.5 pH12 -2699.1 Q0.1*pH4 -2141.2 Q0.5*pH4-1263.4 Q1*pH4 + 1421.3 Q0.1*pH8 +996.7 Q0.5*pH8 + 1174.5 Q1*pH8

\begin{tabular}{lrrrr}
\hline \multicolumn{5}{c}{ b) Remoción de fenoles } \\
\hline Fuente & GL & SC & CM & p \\
\hline quitosano (\%) & 3 & 6066.3 & 2022.11 & 0.000 \\
pH & 2 & 11336.0 & 5667.98 & 0.000 \\
Interacción & 6 & 4312.3 & 718.72 & 0.000 \\
Error & 24 & 78.0 & & \\
Total & 35 & 21792.6 & & \\
\hline
\end{tabular}

R-cuad. $=99.64 \%$

Modelo lineal general. Fenoles vs. quitosano en polvo, pH

Fenoles = 42.9646+ 21.4356 Q0.1 -3.9257 Q0.5 - 3.9673 Q1 - 13.5446 Q1.5 + 19.9627 pH4 + 3.1887 pH8 $+23.1516 \mathbf{p H 1 2}-1.8401 \mathbf{Q 0 . 1}$ * pH4 -6.9116 Q0.5*pH4 + 6.9488 Q1*pH4 + $21.2596 \mathbf{Q 0 . 1}$ * pH8 + 3.3657 Q0.5*pH8 - 10.2103 Q1*pH8

\begin{tabular}{lrccc}
\hline \multicolumn{5}{c}{ c) Remoción de azúcares } \\
\hline Fuente & GL & SC & CM & $\mathrm{p}$ \\
\hline quitosano (\%) & 3 & 0.041969 & 0.0139896 & 0.000 \\
pH & 2 & 0.085499 & 0.0427493 & 0.000 \\
Interacción & 6 & 0.039830 & 0.0066383 & 0.000 \\
Error & 24 & 0.021273 & 0.0008864 & \\
Total & 35 & 0.188570 & & \\
\hline
\end{tabular}

R-cuad. $=88.72 \%$

Modelo lineal general. Azúcares vs. quitosano en polvo, pH

Azúcares $=\mathbf{0 . 2 6 6 7 3 1}+0.023135$ Q0.1 + 0.029610 Q1 + 0.063372 pH4 -0.02931 Q0.1*pH4 + 0.02997 Q0.1*pH8 + 0.03070 Q0.5*pH8 0.01620 Q1*pH8

\section{d) Remoción de ST}

\begin{tabular}{lrrrr}
\hline Fuente & GL & \multicolumn{1}{c}{ SC } & \multicolumn{1}{c}{ CM } & \multicolumn{1}{c}{$\mathrm{p}$} \\
\hline Alginato (\%) & 3 & 65470519 & 21823506 & 0.000 \\
pH & 2 & 655806 & 327903 & 0.000 \\
Interacción & 6 & 2684740 & 447456 & 0.000 \\
Error & 24 & 659171 & 34332 & \\
Total & 35 & 69470236 & & \\
\hline
\end{tabular}

R-cuad. $=98.05 \%$

Modelo lineal general. ST vs. quitosano en polvo, $\mathrm{pH}$

$\mathbf{S T}=\mathbf{2 1 2 0 8 . 1}+138.3 \mathbf{Q 0 . 1}-832.1$ Q0.5 -143.1 Q1 + 129.7 pH4 -132.8 pH8 + 115.1Q0.1*pH4 124.2Q0.5*pH4 + 226.06 Q1*pH4 - 232.1 Q1.5*pH4 + 821.3 Q0.1*pH8 - 141.1 Q1.5*pH8

$\mathrm{DQO}=$ demanda química de oxígeno, $\mathrm{ST}=$ sólidos totales, $\mathrm{GL}=$ grados de libertad, $\mathrm{SC}=$ suma de cuadrados, $\mathrm{CM}=$ cuadrado medio, $\mathrm{p}=$ probabilidad 

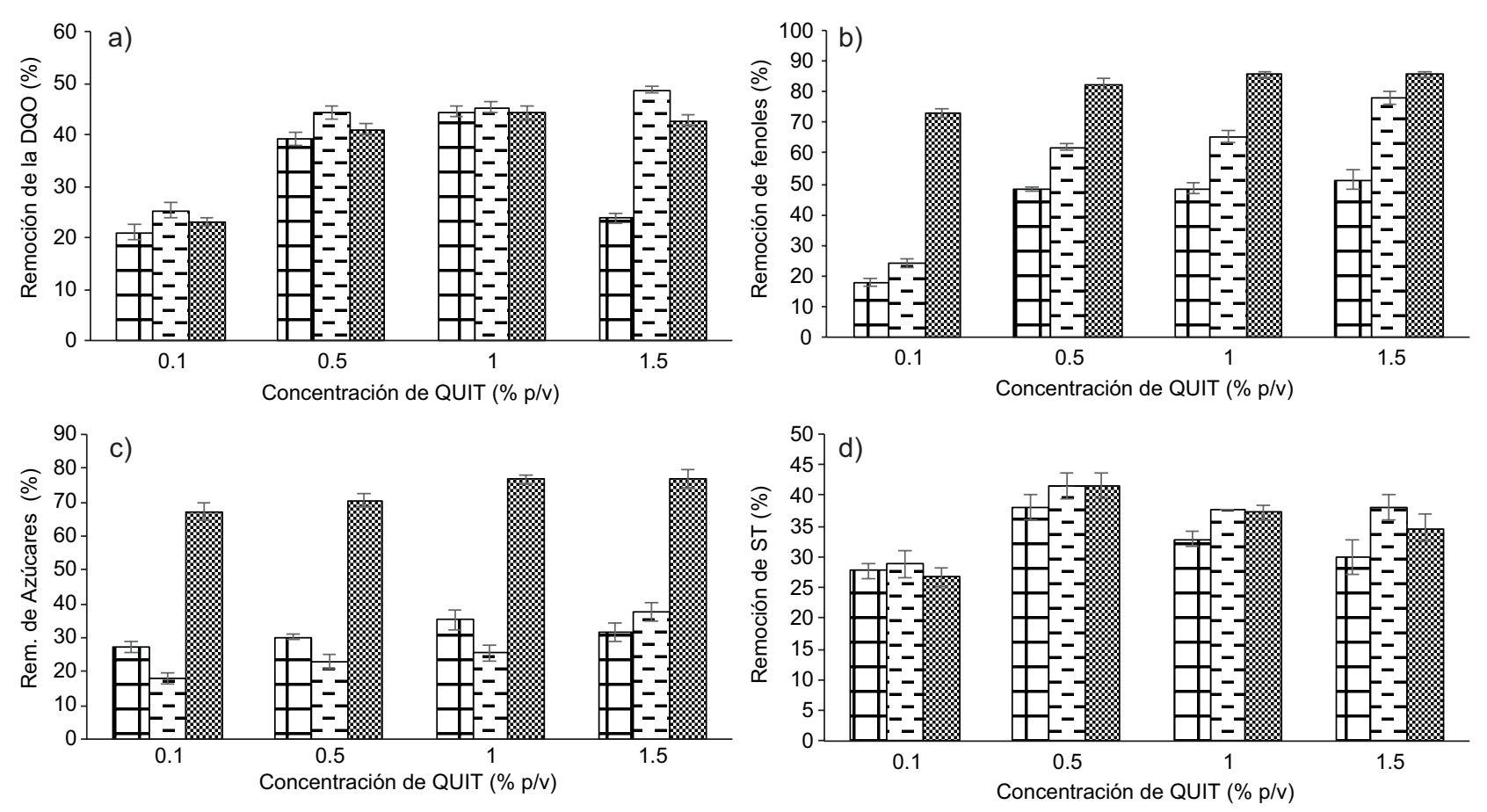

\begin{tabular}{|lll|}
\hline $\mathrm{pH} 4$ & $\square \mathrm{pH} 8$ & $\mathrm{OpH} 12$ \\
\hline
\end{tabular}

Fig. 3. Porcentaje de remoción de contaminantes en función de la concentración de quitosano en polvo: $0.1,0.5,1.0$ y $1.5 \%$ (p/v)) y el pH: 4, 8 y 12. a) demanda química de oxígeno (DQO), b) fenoles, c) azúcares y d) sólidos totales (ST; $\mathrm{p} \leq 0.05)$

Ozkorucuklu et al. (2009) han reportado que los valores de pKa para diferentes ácidos fenólicos se ubican entre 4.20 y 4.40 para el grupo carboxilo y entre 8.70 y 9.40 para el hidroxilo del grupo fenólico. En este sentido es de esperar que al incrementarse el $\mathrm{pH}$ de la solución en que se encuentren los compuestos fenólicos presentes en el nejayote, estos tenderán a incrementar su carga negativa y en consecuencia a unirse a la superficie del quitosano por interacciones electrostáticas (Fig. 3b). En la figura 3b también se puede apreciar que la eficiencia de remoción de los fenoles no varía de manera importante con la concentración de quitosano. Los niveles de remoción que se muestran en esta figura, sugieren que el área de contacto con las moléculas del quitosano tiene un efecto poco importante para la remoción de los fenoles presentes en el nejayote.

Respecto a la remoción de azúcares (Fig. 3c) se observó un comportamiento similar al observado en los fenoles con dos pendientes definidas. La primera ( $\mathrm{pH}$ de 4 a 8) es menos pronunciada que la observada en los fenoles debido a que no existen grupos ionizables en los azúcares del nejayote en este intervalo de $\mathrm{pH}$, y la remoción de estos compuestos varía ligeramente con el $\mathrm{pH}$. En contraste, la última región $(\mathrm{pH}=$ 8 a 12) es más pronunciada debido a la ionización de los grupos hidroxilo de los azúcares que se adsorben al quitosano de manera semejante a como se observó en los fenoles, es decir, por interacciones iónicas.

En relación con la remoción de ST, en la figura $3 \mathrm{~d}$ se muestra que en los tratamientos a $\mathrm{pH} 8$ y $\mathrm{pH} 12$ se logra remover de 37 a $41 \%$, mientras que a $\mathrm{pH} 4$ se alcanza una eficiencia de 28 a $38 \%$. Esto sugiere que a medida que disminuye el $\mathrm{pH}$ la disolución del polvo de quitosano se favorece por la protonación de sus grupos amino, incrementando la concentración de ST en el nejayote, lo cual se refleja en una disminución de la eficiencia de remoción de estos sólidos. Por otra parte, cuando se incrementó la concentración de quitosano en polvo, la hidrólisis de este polisacárido incrementó el contenido de sólidos disueltos. Para comprobar lo antes mencionado se utilizó un testigo, es decir, se dio tratamiento a muestras de agua bajo las mismas condiciones de tratamiento que al nejayote y se determinó la cantidad de ST remanente. Se obtuvo que a valores de $\mathrm{pH} 4$ el quitosano aporta 5.01 $\pm 0.163 \%$ en promedio, a $\mathrm{pH} 8,4.35 \pm 0.114 \%$ y a $\mathrm{pH} 12,4.93 \pm 0.123 \%$. Esto afecta su eficiencia para la remoción de contaminantes del nejayote.

\section{Tratamiento secuencial}

Con el fin de probar la efectividad de la combinación de los polímeros empleados en los tratamientos 
anteriores, se realizó un tratamiento utilizando las condiciones que produjeran los mejores valores en la remoción de la DQO. Se utilizó este parámetro debido a que cualquier incremento en la cantidad de contaminantes orgánicos e inorgánicos se refleja en un aumento de este parámetro. Así, en primera instancia se aplicó el tratamiento con quitosano soluble al $0.8 \%$ al nejayote y posteriormente al efluente resultante de este procedimiento se le trató con solución de alginato al $0.33 \%(\mathrm{p} / \mathrm{v})$ (tratamiento quitosano-alginato). Del mismo modo, para determinar si el orden de la aplicación de ambos biopolímeros afectaba la remoción de contaminantes del nejayote, se realizó el tratamiento a muestras de nejayote a $\mathrm{pH} 4$ con solución de alginato al $0.33 \%$ y enseguida se aplicó al efluente resultante la metodología previamente descrita utilizando quitosano soluble al $0.8 \%(\mathrm{v} / \mathrm{v})$ (tratamiento alginato-quitosano). Del mismo modo, se determinó la eficiencia de remoción de los mismos parámetros utilizados con alginato y quitosano de manera independiente (DQO, fenoles, azúcares y ST).

En el cuadro $\mathbf{V}$ se muestra la eficiencia de remoción de la DQO, fenoles, azúcares y ST al aplicar los biopolímeros de manera independiente (tratamientos quitosano y alginato) y secuencial (quitosano-alginato y alginato-quitosano). Como puede observarse, el tratamiento con alginato tiene mayor eficiencia de remoción de DQO, fenoles y azúcares que el tratamiento con quitosano, pero pasa lo contrario con los ST. Como ya se mencionó anteriormente, el alginato adsorbe moléculas disueltas y atrapa sólidos suspendidos del nejayote, lo que resulta en una mayor remoción. La baja eficiencia en la remoción de ST podría deberse a que en el pretratamiento de la muestra de nejayote se añade un agente secuestrante del $\mathrm{Ca}^{+2}$ (citrato de sodio) y posteriormente, para el proceso de gelificación, se adiciona $\mathrm{CaCl}_{2}$. Ambas sustancias disueltas en la muestra incrementan el contenido de ST y dan como resultado una baja eficiencia de remoción de esta variable de respuesta.

Con respecto a los tratamientos secuenciales, se encontró que el tratamiento alginato-quitosano es más eficiente en la remoción de todos los parámetros de respuesta (\% de remoción de DQO, de fenoles, de azúcares y de ST). El material remanente del tratamiento con alginato (polímero aniónico) soluble y coloidal con carga negativa es atraído por el quitosano (polímero catiónico) a través de interacciones electrostáticas. Esto permite aumentar el porcentaje de remoción de los valores de DQO y fenoles. Sin embargo la remoción de los azúcares no es tan eficiente, probablemente por la hidrólisis del alginato y el quitosano que están constituidos por azúcares. Al tratar la muestra de nejayote con un sólo polímero, resulta ser más eficiente el alginato $(61.43 \%$ remoción de la DQO), pero si éste es seguido de un tratamiento con quitosano (alginato-quitosano) la reducción es mayor (70.19\% de la DQO). Dicho tratamiento mejora la eficiencia en la remoción de contaminantes en un $10 \%$. El análisis de varianza mostró diferencia significativa entre los tratamientos $(\mathrm{p} \leq 0.001)$.

En otros estudios, Ferreira-Rolón et al. (2014) aplicaron un tratamiento anaeróbico al nejayote por medio de un reactor de lodos de flujo ascendente (UASB). Se utilizó agua residual municipal para diluir el nejayote (1-3 g/L de DQO) resultando una remoción del $90 \%$ a una temperatura de $32{ }^{\circ} \mathrm{C}$. En otro estudio, Suárez-Meraz et al. (2016) mostraron la utilidad de remover material coloidal del nejayote con

CUADRO V. PORCENTAJE DE REMOCIÓN DE CONTAMINANTES EN TRATAMIENTO CON QUITOSANO SOLUBLE, ALGINATO Y TRATAMIENTO SECUENCIAL

\begin{tabular}{lcccc}
\hline Tratamiento & DQO $(\%)$ & Fenoles $(\%)$ & Azúcares (\%) & ST $(\%)$ \\
\hline Quitosano & $59.74 \pm 0.36^{\mathrm{a}}$ & $46.56 \pm 2.11^{\mathrm{a}}$ & $55.51 \pm 1.71^{\mathrm{a}}$ & $30.51 \pm 0.85^{\mathrm{a}}$ \\
Alginato & $61.43 \pm 0.24^{\mathrm{b}}$ & $83.46 \pm 1.76^{\mathrm{b}}$ & $60.78 \pm 0.33^{\mathrm{a}}$ & $15.08 \pm 1.95^{\mathrm{b}}$ \\
Quitosano-alginato & $67.21 \pm 0.85^{\mathrm{b}}$ & $55.34 \pm 0.71^{\mathrm{a}}$ & $60.23 \pm 1.06^{\mathrm{a}}$ & $10.47 \pm 1.90^{\mathrm{c}}$ \\
Alginato-quitosano & $70.19 \pm 0.24^{\mathrm{c}}$ & $88.12 \pm 8.82^{\mathrm{b}}$ & $61.37 \pm 0.02^{\mathrm{a}}$ & $46.11 \pm 2.61^{\mathrm{d}}$ \\
\hline
\end{tabular}

Quitosano-alginato $=$ Tratamiento secuencial con quitosano soluble al $0.8 \%(\mathrm{p} / \mathrm{v})$ seguido de alginato de sodio al $0.33 \%(\mathrm{p} / \mathrm{v})$.

Alginato-quitosano $=$ Tratamiento secuencial con quitosano soluble al $0.8 \%(\mathrm{p} / \mathrm{v})$ seguido de alginato de sodio al $0.33 \%(\mathrm{p} / \mathrm{v})$

a b c: Diferencia significativa de acuerdo con la prueba de Tukey $(\mathrm{p} \leq 0.05)$. Medias con letra diferente en cada columna tienen diferencia significativa

\pm Desviación estándar, DQO = demanda química de oxígeno, $\mathrm{ST}=$ sólidos totales 
un tratamiento de coagulación-floculación a muestras de nejayote centrifugado. Utilizaron quitosano de diferentes pesos moleculares y lograron remover más del $80 \%$ de la turbiedad, lo que les permitió cumplir con la norma oficial mexicana NOM-002-SEMARNAT-1996 (SEMARNAT 1996a). A diferencia de los estudios mencionados, en los procesos realizados en este reporte se utilizó nejayote completo. Se utilizaron biopolímeros (alginato y quitosano) que, en primera instancia, no afectan al ambiente ya que son fáciles de degradar. El biopolímero que dio mejores resultados fue el alginato a $\mathrm{pH} 4$ con una concentración del $0.33 \%$, ya que se logró remover el $61 \%$ de la DQO. De esta manera, este tratamiento permite reducir las operaciones unitarias como el centrifugado previo al tratamiento o el mezclado con otro tipo de agua. El agua residual de este tratamiento, si bien no alcanza a cubrir con las especificaciones establecidas por las normas oficiales para descargarla en ríos, sí permite las descargas al suelo para darle uso como riego agrícola o descargarla al alcantarillado urbano o municipal.

\section{CONCLUSIONES}

El tratamiento del nejayote completo con alginato y quitosano por separado resultó efectivo para disminuir los niveles de contaminantes, debido al proceso de atrapamiento de las cadenas de alginato y de los mecanismos de adsorción-precipitación del quitosano. La utilización de alginato a una concentración de $0.33 \%(\mathrm{p} / \mathrm{v})$ y a $\mathrm{pH} 4$ y de quitosano con una concentración del $0.8 \%(\mathrm{v} / \mathrm{v})$ favoreció la disminución de la DQO alcanzando una remoción de $61.43 \%$ y $59.74 \%$ respectivamente. El tratamiento secuencial con alginato-quitosano mejora hasta un $10 \%$ la remoción de la DQO respecto a los tratamientos con los biopolímeros por separado.

El material suspendido y disuelto del nejayote, al interaccionar con los biopolímeros, reduce de manera significativa la cantidad de los contaminantes (DQO, fenoles, azúcares reductores y ST). Si sólo se lleva a cabo una filtración estos parámetros se reducen en un $33.4 \pm 0.41 \%$ de DQO, $35.2 \pm 0.2 \%$ de fenoles, $34.73 \pm 0.23$ de azúcares y $13 \%$ de ST, mientras que si se utilizan los polímeros de forma secuencial (alginato-quitosano) se disminuye la DQO en un $36 \%$, los fenoles son removidos hasta en un $53 \%$ y los azúcares y ST logran removerse de la fase acuosa hasta en un 24 y $33 \%$, respectivamente. Además, la eficiencia en la reducción de dichos parámetros, facilita el manejo de este tipo de aguas para la eliminación del material sólido del nejayote tratado. Lo anterior sugiere que el quitosano y el alginato son una opción viable para aplicarse en el tratamiento del nejayote debido a que son polisacáridos biodegradables y los residuos sólidos también pueden ser biodegradados de manera efectiva. Por lo anterior, se concluye que estos polímeros tienen potencial para escalarse a nivel industrial, ya que logran disminuir de manera considerable los contaminantes evaluados, lo que le permite obtener un efluente apto para uso en riego agrícola o bien, para descargarlo directamente al drenaje urbano o municipal.

\section{AGRADECIMIENTOS}

A la Dra. Magdalena Samantha Ramos Gómez, Miguel Figueroa Aguilar y José Juan Salinas por el apoyo técnico. Se agradece al Consejo Nacional de Ciencia y Tecnología por el apoyo de la beca para la estudiante de doctorado (No 97268) y a la Universidad Autónoma de Aguascalientes por el apoyo económico para la realización de la tesis.

\section{REFERENCIAS}

Alvarado-Granados A.R., Díaz-Cuenca E. y Guerrero-Peñuelas A. G. (2013). Diagnóstico para el saneamiento del agua en las cuencas de la laguna de San Miguel Almaya en el Valle de Toluca, México. Quivera 15 (2013-2), 93-121.

APHA, AWWA, WEF (1998). Standard methods for the examination of water and wastewater (20 ed.). American Public Health Association/American Waste Works Association/Water Environmental Federation, Washington, D.C., EUA, 1207 pp.

Argun M. S. y Argum M.E. (2017). Treatment and alternative usage possibilities of special wastewater: nejayote. J. Food Process Eng. 41 (1), 1-7. https://doi. org. 10.1111/jfpe. 12609

Ayala-Soto F. E., Serna-Saldívar S. O., García-Lara S. y Pérez-Carrillo E. (2014). Hydroxycinnamic acids, sugar composition and antioxidant capacity of arabinoxilans extracted from different maize fiber sources. Food Hydrocolloid 35, 471-475. https://doi. org.10.1016/j.foodhyd.2013.07.004

Blackburn R. (2004) Natural polysaccharides and their interactions with dye molecules: applications in effluent treatment. Environ. Sci. Technol. 38 (18), 4905-4909. https://doi.org.10.1021/es049972n 
Berlanga-Reyes C., Carvajal-Millan E., Niño-Medina G., Rascón-Chu A., Ramírez-Wong B. y Magaña-Barajas E. (2011). Low-value maize and wheat by-products as a source of ferulated arabinoxylans. En: Waste water-treatment and reutilization. (F.S. GarcíaEinschlag, Ed.). Intech, Croacia, pp. 341-353. https:// doi.org. 10.5772/15840

Bolto, B. y Gregory J. (2007). Organic polyelectrolytes in water treatment. Water Res. 41 (11), 2301-2324. https:// doi.org.10.1016/j.watres.2007.03.012

Bradford M. M. (1976). A rapid and sensitive method for the quantization of micro-gram quantities of protein utilizing the principle of protein dye binding. Anal. Biochem. 72(1-2), 248-254. https://doi. org.10.1016/0003-2697(76)90527-3

Caldera Y., Rodríguez Y., Oñate H., Prato J. y Gutiérrez E. (2011). Eficiencia del quitosano como coagulante durante el tratamiento de aguas de baja turbidez asociadas a la producción de petróleo. Revista Tecnocientífica URU 1 (1), 45-52.

Campechano-Carrera E. M., Figueroa-Cárdenas J., Arámbula-Villa G., Martínez-Flores H. D., JiménezSandoval S. J. y Luna-Bárcenas J. G. (2012). New ecological nixtamalization process for tortilla production and its impact on the chemical properties of whole corn flour and wastewater effluents. Int. J. Food Sci. Tech. 47 (3), 564- 571. https://doi.org.10.1111/j.13652621.2011.02878.x

Castro-Muñoz R., Cerón-Montes G. I., Barragán-Huerta B. E. y Yañez-Fernández J. (2015). Recovery of carbohydrates from nixtamalization wastewaters (nejayote) by ultrafiltration. Rev. Mex. Ing. Quim. 14 (3), 735-744.

Cheng W.P., Chi F.H., Yu R. F. y Lee Y.C. (2005). Using chitosan as a coagulant in recovery of organic matters from the mash and lauter wastewater of brewery. J. Polym. Environ. 13 (4), 383-388. https://doi. org. $10.1007 / \mathrm{s} 10924-005-5533-0$

Core R. T. R (2013). A language and environment for statistical computing. R, Foundation for Statistical Computing, Viena, Austria, 2673 pp.

Donati I. y Paoletti S. (2009). Material properties of alginates. En: Alginates: biology and applications. Vol. 13. (B.H.A. Rehm, Ed.). Springer, Berlín, Alemania, pp. 1-54. https://doi.org.10.1007/978-3-540-92679-5_1

Donati I., Holtan, S., Morch A., Borgogna M., Dentini M. y Skjak-Braek G. (2005). New hypothesis on the role of alternating sequences in calcium-alginate gels. Biomacromolecules 6, 1031-1040. https://doi. org.10.1007/978-3-540-92679-5_1
Durán-de Bazúa C., Sánchez-Tovar S.A., HernándezMorales M.R. y Bernal-González M. (2007). Use of anaerobic-aerobic treatment system for maize processing installations: applied microbiology in Action. Formatex, 3-12

Ferreira-Rolón A., Ramírez-Romero G. y Ramírez-Vives F. (2014). Aumento de la actividad metanogénica en lodos granulares precipitando calcio en el nejayote mediante el burbujeo de $\mathrm{CO}_{2}$. Rev. Mex. Ing. Quim. 13 (2), 517-525.

García-Depraect O., Gómez-Romero J. y León-Becerril E. (2017). A novel biohydrogen production process: co-digestion of vinasse and nejayote as complex raw substrates using a robust inoculum. Int. J. Hydrogen. Energ. 42 (9), 5820-583. https://doi.org.10.1016/j. ijhydene.2016.11.204

García-Zamora J. L., Sánchez-González M., Lozano, J.A., Jáuregui J., Zayas T., Santacruz V., Hernández F. y Torres E. (2015). Enzymatic treatment of wastewater from the corn tortilla industry using chitosan as an adsorbent reduces the chemical oxygen demand and ferulic acid content. Process Biochem. 50, 125-133. https://doi.org.10.1016/j.procbio.2014.10.012

Ge Y., Cui X., Liao Ch. y Li Z. (2017). Facile fabrication of green geopolymer/alginate hybrid spheres for efficient removal of $\mathrm{Cu}$ (II) in water: Batch and column studies. Chem. Eng. J. 311, 126-134. https://doi.org.10.1016/j. cej.2016.11.079

Guerrero L., Omil F., Méndez R. y Lema J. M. (1998). Protein recovery during the overall treatment of wastewaters from fish-meal factories. 63 (3). 221-229. Bioresour Technol. https://doi.org.10.1016/S09608524(97)00140-5

Guibal E., Van Vooren M., Dempsey B.A. y Roussy J. (2006). A review of the use of chitosan for the removal of particulate and dissolved contaminants. Sep. Sci. Technol. 41, 2487-2514. https://doi. org. 10.1080/01496390600742807

Guibal E. y Roussy J. (2007). Coagulation and flocculation of dye-containing solutions using a biopolymer (Chitosan). React. Funct. Polym. 67, 33-42. https://doi. org.10.1016/j.reactfunctpolym.2006.08.008

Gutiérrez-Cortéz E., Rojas-Molina I., Rojas A., Arjona J.L., Cornejo-Villegas M.A., Zepeda-Benítez Y., Velázquez-Hernández R., Ibarra-Alvarado C. y Rodríguez-García M.E. (2010). Microstructural changes in the maize kernel pericarp during cooking stage in nixtamalization process. J. Cereal Sci. 51, 8188. https://doi.org.10.1016/j.jcs.2009.09.008 
Gutiérrez-Uribe J. A., Rojas-García C., García-Lara S. y Serna-Saldívar S. O. (2010). Phytochemical analysis of wastewater (nejayote) obtained after lime-cooking of different types of maize kernels processed into masa for tortillas. J. Cereal Sci. 52, 410-416. https://doi. org. 10.1016/j.jcs.2010.07.003

Janve B., Yang W., Kozman A., Sims C., Teixeira A., Gunderson M. A. y Rababah T. M. (2013). Enhancement of corn nixtamalization by power ultrasound. Food Bioprocess Technol. 6, 1269-1280. https://doi. org. 10.1007/s11947-012-0816-7

Jáuregui-Rincón J., Lozano-Álvarez J. A. y MedinaRamírez I. (2011). Zimm-Bragg model applied to sorption of dyes by biopolymers: alginic acid and xanthan. En: Biotechnology of Biopolymers (M. Elnashar, Ed.) 9, 165-190. https://doi.org.10.5772/17074

Jia S., Yang Z., Yang W., Zhang T., Zhang S., Yang X., Dong Y., Wu Y. y Wang Y. (2016). Removal of Cu(II) and tetracycline using an aromatic rings-functionalized chitosan-based flocculant: Enhanced interaction between the flocculant and the antibiotic. Chem. Eng. J. 283, 495-503. https://doi.org.10.1016/j.cej.2015.08.003

Li J., Song X., Pan J., Zhong L., Jiao S. y Ma Q. (2013). Adsorption and flocculation of bentonite by chitosan with varying degree of deacetylation and molecular weight. Int. J. Biol. Macromol. 62, 4-12. https://doi. org.10.1016/j.ijbiomac.2013.08.009

Lin N., Bruzzese C. y Dufresne A. (2012). Tempo-oxidized nanocellulose participating as crosslinking aid for alginate-based sponges. ACS Applied Materials and Interfaces 4 (9), 4948-4959. https://doi.org.10.1021/ am301325r

Lozano-Álvarez J. A., Marañón-Ruiz V.F., JáureguiRincón J., Medina-Ramírez I., Frausto-Reyes C. y Salinas-Gutiérrez R. (2015) Removal of direct dyes with alginic acid. J. Mex. Chem. Soc. 59, 215-228. https://doi.org.10.29356/jmcs.v59i3.38

Lozano-Álvarez J.A., Jáuregui-Rincón J., Mendoza-Díaz G., Rodríguez-Vázquez R. y Frausto-Reyes C. (2009). Study of sorption equilibrium of biopolymers alginic acid and xanthan with C.I. disperse yellow 54. J. Mex. Chem. Soc. 53, 59-70. https://doi.org.10.29356/jmcs. v53i2.1007

Maurstad, G., Danielsen S. y Stokke B.T. (2003). Analysis of compacted semiflexible polyanion visualized by atomic force microscopy: influence of chain stiffness on the morphologies of polyelectrolyte complexes. J. Phys. Chem. 107, 8172-8180. https://doi.org.10.1021/ jp0271965
Méndez-Albores A., Zamora-Rodríguez D., ArámbulaVilla G., Vázquez-Duran A. y Moreno-Martínez E. (2014). Impact of different alkaline-heating processes on technological and nutritional properties of maize tortillas. J. Food Nutr. Res. 53, 60-70.

Meng H., Zheng j., Wen X., Cai Z., Zhang J. y Chen T. (2015). pH-and Sugar-Induced shape memory hydrogel based on reversible phenoylboronic acid-diol ester bonds. Macromol. Rapid Commun. 36, 533-537. https://doi.org.10.1002/marc.201400648

Miller G. L. (1959). Use of dinitrosalicylic acid reagent for determination of reducing sugar. Anal. Chem. 31 (3), 426-428. https://doi.org.10.1021/ac60147a030

Ozkorucuklu S.P., Beltrán J.L., Fonrodona G., Barrón D., Alsancak G. y Barbosa J. (2009). Determination of dissociation constants of some hydroxylated benzoic and cinnamic acids in water from mobility and spectroscopic data obtained by CE-DAD. J. Chem. Eng. Data. 54, 807-811. https://doi.org.10.1021/ je $800595 \mathrm{x}$

Paredes-López O., Guevara-Lara F. y Bello-Pérez L. (2009). La nixtamalización y el valor nutritivo del maíz. Ciencias 92, 60-70.

Perju M.M. y Dragan E.S. (2010). Removal of azo dyes from aqueous solutions using chitosan based composite hydrogels. Ion Ex. Lett. 3, 7-11. https://doi. org.10.3260/iel.2010.06.002

Pinotti A., Bevilacqua A. y Zaritzky N. (1997). Optimization of the flocculation stage in a model system of a food emulsion waste using chitosan as polyelectrolyte. J. Food Eng. 32 (1), 69-81. https://doi.org.10.1016/ S0260-8774(97)00003-4

Pulido R., Escárcega C. y Durán de Bazúa C. (1987). Modelo cinético para reactores biológicos rotatorios usados en el tratamiento aerobio de efluentes líquidos de industria del maíz. Tecnología Ciencia y Educación 2 (1), 15-24.

Quin Y., Cai L., Feng D., Shi B., Liu J., Zhang W. y Shen Y. (2007). Combined use of chitosan and alginate in the treatment of wastewater. J. Appl. Polym. Sci. 104, 3581-3587. https://doi.org.10.1002/app.26006

Ramírez-Romero G., Reyes-Velázquez M. y Cruz-Guerrero A. (2013). Estudio del nejayote como medio de crecimiento de probióticos y producción de bacteriocinas. Rev. Mex. Ing. Quím. 12 (3), 463-471.

Renault F., Sancey B., Badot P. y Crini G. (2009). Chitosan for coagulation/flocculation processes - An eco-friendly approach. Eur. Polym. J. 45, 1337-1348. https://doi.org.10.1016/j.eurpolymj.2008.12.027 
Retes-Pruneda J. L., Dávila-Vázquez G., Medina-Ramírez I., Chávez-Vela N.A., Lozano-Álvarez J. A., AlatristeMondragón, F. y Jáuregui-Rincón J. (2014). High removal of chemical and biochemical oxygen demand from tequila vinasses by using physicochemical and biological methods. Environ. Technol. 35 (13-16), 1-12. https://doi.org.10.1080/09593330.2014.882960

Roussy R., van Vooren M.V. y Guibal G. (2005). Treatment of ink-containing wastewater by coagulation/flocculation using biopolymers. Water SA 31 (3), 369-376. https://doi.org.10.4314/wsa.v31i3.5208

Roussy J., van Vooren M., Dempsey B.A. y Guibal E. (2005). Influence of chitosan characteristics on the coagulation and the flocculation of bentonite suspensions. Water Res. 39 (14), 3247-3258. https://doi. org.10.1016/j.watres.2005.05.039

Rojas-García C., García-Lara S., Serna-Saldívar S. O. y Gutiérrez-Uribe J. A. (2012). Chemopreventive effects of free and bound phenolics associated to steep waters (nejayote) obtained after nixtamalization of different maize types. Plant Foods Hum. Nutr. 67, 94-99. https:// doi.org.10.1007/s11130-011-0272-y

Ruiz-Gutiérrez M.G., Quintero-Ramos A., MeléndezPizarro C.O., Lardizábal-Gutiérrez D., Barnard J., Márquez-Meléndez R. y Talamás-Abbud R. (2010). Changes in mass transfer, thermal and physicochemical properties during nixtamalization of corn with and without agitation at different temperatures. J. Food Eng. 98, 76-83. https://doi.org.10.1016/j. jfoodeng.2009.12.010

Salazar R., Arámbula-Villa G., Luna-Bárcenas G., Figueroa-Cárdenas J.D., Azuara E. y Vázquez-Landaverde P.A. (2014). Effect of added calcium hydroxide during corn nixtamalization on acrylamide content in tortilla chips. LWT - Food Sci. Technol. 56, 87-92. https://doi.org.10.1016/j.lwt.2013.10.046

Saleha A., Ibrahimb A., Abdelhaib F., Elsharmaa E., Metwallya E. y Siyam T. (2017). Preparation of poly(chitosan-acrylamide) flocculant using gamma radiation for adsorption of $\mathrm{Cu}$ (II) and $\mathrm{Ni}$ (II) ions. Radiat. Phys. Chem. 134, 33-39. https://doi.org.10.1016/j. radphyschem.2017.01.019

Sahu O. y Chaudhari P.K. (2013). Review on chemical treatment of industrial waste water. J. Appl. Sci. Environ. Manage. 17 (2), 241-257. https://doi. org.10.4314/jasem.v17i2.8

Salmerón-Alcocer A., Rodríguez-Mendoza N., PinedaSantiago S., Cristiani-Urbina E., Juárez-Ramírez C., Ruíz-Ordaz N. y Galíndez-Mayer J. (2003). Aerobic treatment of maize-processing wastewater (nejayote) in a single stream multistage reactor. J. Environ. Eng. Sci. 2, 401-406. https://doi.org.1139/s03-046

SEPAFIN (1978). Norma Oficial Mexicana NMX-F090-S-1978. Determinación de fibra cruda en alimentos. Secretaría de Patrimonio y Fomento Industrial. Diario Oficial de la Federación. 27 de marzo de 1979.

SCFI (2001). Norma Oficial Mexicana NMX-AA028-SCFI-2001. Análisis de agua-determinación de la demanda bioquímica de oxígeno en aguas naturales, residuales $\left(\mathrm{DBO}_{5}\right)$ y residuales tratadas - método de prueba. Secretaría de Comercio y Fomento Industrial. Diario Oficial de la Federación. 17 de abril de 2001.

SCFI (2001a). Norma Oficial Mexicana NMX-AA050-SCFI-2001. Análisis de agua - determinación de fenoles totales en aguas naturales, potables, residuales y residuales tratadas - método de prueba. Secretaría de Comercio y Fomento Industrial. 1 de agosto de 2001. SCFI (2001b). Norma Oficial Mexicana NMX-AA072-SCFI-2001. Análisis de agua - determinación de dureza total en aguas naturales, residuales y residuales tratadas - método de prueba. Secretaría de Comercio y Fomento Industrial. Diario Oficial de la Federación. 14 de agosto de 2001.

SCFI (2012). Norma Oficial Mexicana NMX-AA-030/1SCFI-2012. Análisis de agua - medición de la demanda química de oxígeno en aguas naturales, residuales y residuales tratadas- método de prueba - parte 1 método de reflujo abierto. Secretaría de Comercio y Fomento Industrial. Diario Oficial de la Federación. 21 de mayo de 2013.

SCFI (2013). Norma Oficial Mexicana NMX-AA004-SCFI-2013. Análisis de agua - determinación de sólidos sedimentables en aguas naturales, residuales y residuales tratadas - método de prueba. Secretaría de Comercio y Fomento Industrial. Diario Oficial de la Federación. 13 de noviembre de 2013.

SEMARNAT (1996). Norma Oficial Mexicana NOM001-SEMARNAT-1996 (1996). Establece los límites máximos permisibles de contaminantes en las descargas de aguas residuales en aguas y bienes nacionales. Secretaría del Medio Ambiente y Recursos Naturales. Diario Oficial. 6 de enero de 1997.

SEMARNAT (1996a). Norma Oficial Mexicana NOM002-SEMARNAT-1996. Que establece los límites máximos permisibles de contaminantes en las descargas de aguas residuales a los sistemas de alcantarillado urbano o municipal. Secretaría del Medio Ambiente y Recursos Naturales. Diario Oficial. 3 de junio de 1998. 
Singh R. P., Tripathy T., Karmakar G.P., Rath S. K., Karmkar N. C., Pandey S. R., Kannan K., Jain S. K. y Lan N. T. (2000). Novel biodegradable flocculant based on polysaccharides. Current Science 78 (7), 798-803.

Skwarek E., Goncharuk O., Sternik D., Janusz W., Gdula K. y Gun'ko V. (2017). Synthesis, structural, and adsorption properties and thermal stability of nanohydroxyapatite/ polysaccharide composites. Nanoscale Res. Lett. 155, 2-12. https://doi.org.10.1186/s11671-017-1911-5

Suárez-Meraz K.A., Ponce-Vargas S.M., López-Maldonado J.T., Cornejo-Bravo J.M., Oropeza-Guzmán M.T. y López-Maldonado E.A. (2016). Eco-friendly innovation for nejayote coagulation-flocculation process using chitosan: Evaluation through zeta potential measurements. Chem. Eng. J. 284, 536-542. https:// doi.org.10.1016/j.cej.2015.09.026

Tao X., Li A. y Yang H. (2017). Immobilization of metals in contaminated soils using natural polymer-based stabilizers. Environ. Pollut. 222, 348-355. https://doi. org.10.1016/j.envpol.2016.12.028

Valderrama-Bravo C., Gutiérrez-Cortez E., ContrerasPadilla M., Rojas Molina I., Mosquera J. C., Rojas-
Molina A., Beristain F. y Rodríguez-García M. E. (2012). Constant pressure filtration of lime water (nejayote) used to cook kernels in maize processing. J. Food Eng. 110, 428-486. https://doi.org.10.1016/j. jfoodeng.2011.12.018

Wang Q.Z., Chen X.G., Liu N., Wang S.X., Liu C.S., Meng X.H. y Liu C.G. (2006) Protonation constants of chitosan with different molecular weight and degree of deacetylation. Carbohydr. Polym. 65, 194-201. https:// doi.org.10.1016/j.carbpol.2006.01.001

Yan Y., An Q., Xiao Z., Zheng W. y Zhai S. (2017). Flexible core-shell/bead-like alginate@PEI with exceptional adsorption capacity, recycling performance toward batch and column sorption of $\mathrm{Cr}(\mathrm{VI})$. Chem. Eng. J. 313, 475-486. https://doi.org.10.1016/j. cej.2016.12.099

Zhuo N., Lan Y., Yang W., Yang Z., Li X., Zhou X., Liu Y., Shen J. y Zhang X. (2017). Adsorption of three selected pharmaceuticals and personal care products (PPCPs) onto MIL-101(Cr)/natural polymer composite beads. Sep. Purif. Technol. 177, 272-280. https://doi. org.10.1016/j.seppur.2016.12.041 